\title{
Generalized Lamé functions. I. The elliptic case
}

\author{
S. N. M. Ruijsenaars \\ Centre for Mathematics and Computer Science, P.O. Box 94079, \\ 1090 GB Amsterdam, The Netherlands
}

(Received 24 April 1998; accepted for publication 17 September 1998)

\begin{abstract}
We present and study a class of functions associated with the two-particle quantum relativistic Calogero-Moser system with elliptic interactions. The functions may be viewed as joint eigenfunctions of two independent commuting analytic difference operators, one of which is the defining quantum dynamics; The second one is obtained by interchanging the step size and the imaginary period. The functions depend on parameters that are dense in the natural parameter domain. In essence, they consist of products of Weierstrass $\sigma$-functions and plane waves. The zeros of the $\sigma$-functions satisfy a constraint system encoding both Schrödinger equations at once. (C) 1999 American Institute of Physics. [S0022-2488(99)02402-0]
\end{abstract}

\section{INTRODUCTION}

This paper is concerned with eigenfunctions of an analytic difference operator generalizing the Lamé differential operator,

$$
H_{\mathrm{nr}}=-\frac{d^{2}}{d x^{2}}+g(g-1) \wp(x)
$$

where $\wp$ is the Weierstrass $\wp$-function. The pertinent analytic difference operator (henceforth $\mathrm{A} \Delta \mathrm{O}$ ) can be taken to be

$$
H_{\mathrm{rel}}=\left(\frac{\sigma(x-i \beta g)}{\sigma(x)}\right)^{1 / 2} T_{i \beta}\left(\frac{\sigma(x+i \beta g)}{\sigma(x)}\right)^{1 / 2}+(\beta \rightarrow-\beta),
$$

where $\sigma$ is the Weierstrass $\sigma$-function. Here, the shift operator $T_{\alpha}$ is defined by

$$
\left(T_{\alpha} f\right)(x)=f(x-\alpha), \quad \alpha \in \mathrm{C},
$$

so that one has

$$
H_{\mathrm{rel}}=2+\beta^{2} H_{\mathrm{nr}}+O\left(\beta^{4}\right), \quad \beta \rightarrow 0 .
$$

The subscripts " $n r$ " and "rel" in these formulas stand for "nonrelativistic" and "relativistic." Indeed, the parameter $\beta$ in the $\mathrm{A} \Delta \mathrm{O}(1.2)$ may be viewed as $1 / c$, with $c$ the speed of light. Thus, (1.4) encodes the nonrelativistic limit $c \rightarrow \infty$; cf. Ref. 1 .

In our survey paper, Ref. 2 , and lecture notes, Ref. 3, we have announced and described $H_{\text {rel }}$ eigenfunctions for integer coupling $g$. These functions generalize the $H_{\mathrm{nr}}$ eigenfunctions for integer $g$ in the form presented on pp. 572-574 of Ref. 4. In this paper we shall not only elaborate on the $g=2,3, \ldots H_{\text {rel }}$ eigenfunctions from Subsection 6.3 in Ref. 3, but also obtain eigenfunctions for a dense set in the relevant parameter space. As we will detail, these functions are, in fact, joint eigenfunctions of three commuting independent $\mathrm{A} \Delta \mathrm{O}$ s-a feature that generalizes symmetry properties of the hyperbolic specialization described in Subsection 6.3 of Ref. 3. (In the elliptic case, however, we were unable to find useful "dual operators"- -operators $D$ acting on the spectral variable in the eigenfunctions in such a way that the latter are also $D$-eigenfunctions with $x$-dependent eigenvalues.) 
In this paper we shall not dwell on the integrable system context in which the above operators arise. This setting is discussed at length in Refs. 2, 3, and is not necessary for understanding the following. On the other hand, the ancestry of the operators at issue makes itself felt in the aspects we emphasize: We are principally interested in real eigenvalues, and, more generally, in those features of the eigenfunctions that are important in promoting them to kernels of unitary operators that serve to redefine the $\mathrm{A} \Delta \mathrm{O}$ s involved as bonafide self-adjoint Hilbert space operators. In this connection we recall that $\mathrm{A} \Delta \mathrm{O}$ s have highly nonunique eigenfunctions (compared to differential and discrete difference operators), so that quite novel problems and features arise in their rigorous definition as quantum dynamics.

Next, we mention that a close relative $S_{0}$ of the $\mathrm{A} \Delta \mathrm{O} H_{\text {rel }}$ was already introduced by Sklyanin, ${ }^{5,6}$ together with $\mathrm{A} \Delta \mathrm{O}$ s of a similar form. He obtained finite-dimensional representation spaces for the resulting A $\Delta \mathrm{O}$ algebra, spanned by very special eigenfunctions of $S_{0}$. General integer- $g$ eigenfunctions of $S_{0}$ were recently presented in a related context by Krichever and Zabrodin. ${ }^{7}$ (Roughly speaking, Sklyanin's functions correspond to eigenfunctions at the band edges in the finite-gap integration picture expounded in Ref. 7-a viewpoint that is far removed from our concerns in this paper.) For $g>2$ these functions have a rather different appearance from the eigenfunctions already detailed in Ref. 3.

Subsequent to Refs. 3 and 7, Felder and Varchenko obtained integer- $g$ eigenfunctions in a form substantially equivalent to ours. They arrived at these eigenfunctions via their comprehensive study of representations of elliptic quantum groups, tying them in with the "algebraic Bethe Ansatz" of the Russian school, and with Baxter's work on the $X Y Z$ model. Their workinasmuch as it concerns the operator $H_{\text {rel }}$ and its eigenfunctions ${ }^{8,9}$ - has a quite different perspective, emphasizing representation theoretic and algebro-geometric features. (See also a recent paper by Hasegawa ${ }^{10}$ for yet another approach.)

Before summarizing our results, we would like to mention three forthcoming papers that are closely related to the present one. First, we point out that hyperbolic and trigonometric specializations are studied in a sequel to this paper..$^{11}$ In the latter regimes we can proceed much further, since a second, far more explicit representation of the relevant eigenfunctions exists. The results obtained in these special contexts also illuminate various issues pertaining to the elliptic regime, to which we restrict attention in this paper.

Second, the simplest nontrivial parameter choice $g=2$ will be reconsidered elsewhere. ${ }^{12}$ This case admits an in-depth treatment that is independent of (and considerably simpler than) the present paper and its sequel. Moreover, as a striking feature of this special case we demonstrate that in a certain scaling limit its eigenfunctions give rise to the well-known eigenfunctions of the quantized nonlinear Schrödinger equation (alias the delta-function gas).

Third, our forthcoming conference contribution Ref. 13 reviews our findings regarding generalized Lamé functions and their specializations.

In order to sketch the results of the present paper, it is expedient to trade the Weierstrass $\sigma$-function $\sigma\left(z ; \omega, \omega^{\prime}\right)$ for the function

$$
s(r, a ; z) \equiv \sigma\left(z ; \frac{\pi}{2 r}, \frac{i a}{2}\right) \exp \left(-\eta z^{2} r / \pi\right)
$$

(Here and below, we use the elliptic function notation of Whittaker and Watson; ${ }^{4}$ we also use some of the elliptic function lore collected in this reference.) The function $s(z)$ is an entire odd function with simple zeros in the lattice points $\mathbb{Z} \pi / r+i \mathbb{Z} a$. It is $\pi / r$-antiperiodic and obeys the analytic difference equation (henceforth $\mathrm{A} \Delta \mathrm{E}$ )

$$
\frac{s(z+i a / 2)}{s(z-i a / 2)}=-\exp (-2 i r z)
$$




$$
\begin{gathered}
\lim _{a \rightarrow \infty} s(r, a ; z)=\frac{\sin r z}{r} \quad \text { (uniformly on compacts), } \\
\lim _{r \rightarrow 0} s(r, a ; z)=\frac{\sinh \pi z / a}{\pi / a} \quad \text { (uniformly on compacts), }
\end{gathered}
$$

and the scaling relation

$$
s(r / \lambda, \lambda a ; \lambda z)=\lambda s(r, a ; z) .
$$

For later use we also note that iteration of the $\mathrm{A} \Delta \mathrm{E}$ (1.6) yields

$$
\frac{s(r, a ; z+i L a)}{s(r, a ; z)}=(-)^{L} \exp \left(a r L^{2}-2 i r L z\right), \quad L \in \mathbb{Z} .
$$

As a matter of fact, we have occasion to use two $s$-functions,

$$
s_{\delta}(z) \equiv s\left(r, a_{\delta} ; z\right), \quad \delta=+,-.
$$

This is because the functions we define and study are actually joint eigenfunctions of the two A $\Delta$ Os

$$
H_{\delta} \equiv e^{-b r}\left(\frac{s_{\delta}(x-i b)}{s_{\delta}(x)}\right)^{1 / 2} T_{i a_{-\delta}}\left(\frac{s_{\delta}(x+i b)}{s_{\delta}(x)}\right)^{1 / 2}+(i \rightarrow-i), \quad \delta=+,-
$$

In view of (1.5), each of these may be regarded as a multiple of the $\mathrm{A} \Delta \mathrm{O} H_{\text {rel }}$ (1.2) when one sets $b=a_{-\delta} g$. The constant up front is chosen such that we have the symmetry property

$$
H_{\delta}\left(a_{+}, a_{-}, b\right)=H_{\delta}\left(a_{+}, a_{-}, a_{+}+a_{-}-b\right) .
$$

[Use (1.6) to verify this.] Here and below, it is understood that the parameters belong to the elliptic parameter domain

$$
\mathcal{E} \equiv\left\{\left(r, a_{+}, a_{-}, b\right) \mid r, a_{+}, a_{-}>0, b \in \mathbb{R}\right\} .
$$

We begin by transforming $H_{\delta}$ to the form

$$
A_{\delta}=e^{-b r} \frac{s_{\delta}(x-i b)}{s_{\delta}(x)} T_{i a_{-\delta}}+(i \rightarrow-i), \quad \delta=+,-,
$$

where

$$
A_{\delta} \equiv w(x)^{-1 / 2} H_{\delta} w(x)^{1 / 2}
$$

The weight function $w\left(r, a_{+}, a_{-}, b ; x\right)$ occurring here was introduced and studied in Ref. 14. It is a meromorphic solution to the two A $\Delta$ Es

$$
\frac{w\left(x+i a_{\delta} / 2\right)}{w\left(x-i a_{\delta} / 2\right)}=\frac{s_{-\delta}\left(x+i b-i a_{\delta} / 2\right)}{s_{-\delta}\left(x-i b+i a_{\delta} / 2\right)} \cdot \frac{s_{-\delta}\left(x+i a_{\delta} / 2\right)}{s_{-\delta}\left(x-i a_{\delta} / 2\right)}, \quad \delta=+,-,
$$

which is why (1.16) entails (1.15).

The point of the similarity transformation (1.16) is that the $\mathrm{A} \Delta \mathrm{Os} A_{\delta}(1.15)$ have meromorphic coefficients. Thus, we may and will view them first as linear operators leaving the vector space

$$
\mathcal{M} \equiv\{F(x) \mid F \text { meromorphic }\}
$$


invariant. (We shall discuss Hilbert space aspects shortly.) In view of the $\mathrm{A} \Delta \mathrm{E}$ (1.6), the maps $A_{+}, A_{-}: \mathcal{M} \rightarrow \mathcal{M}$ commute. Now at this point it should be emphasized that there are a great many functionally independent $\mathrm{A} \Delta \mathrm{O}$ s commuting with $A_{+}$. (For instance, when one multiplies the two coefficients of $A_{-}$by distinct meromorphic functions with period $i a_{-}$, one obtains an $\mathrm{A} \Delta \mathrm{O}$ that also commutes with $A_{+}$.) However, we are not aware of any general arguments guaranteeing the existence of nontrivial joint eigenspaces for $A_{+}$and an independent $\mathrm{A} \Delta \mathrm{O}$ in its commutant.

Even so, we have found two linearly independent joint eigenfunctions $\Psi( \pm x, y)$ of the $\left(a_{+} \leftrightarrow a_{-}\right)$-symmetric pair $\left(A_{+}, A_{-}\right)$, provided the parameters belong to a dense subset $\mathcal{D}$ of the parameter space $\mathcal{E}(1.14)$. (Note both $\mathrm{A} \Delta \mathrm{Os}$ commute with parity.) The spectral variable $y$ takes values in an interval $(K, \infty)$, where $K$ depends on the parameters. The eigenvalues $E_{+}$and $E_{-}$are real-valued, real-analytic functions, satisfying

$$
E_{\delta}(y) \sim \exp \left(a_{-\delta} y\right), \quad E_{\delta}^{\prime}(y) \sim a_{-\delta} \exp \left(a_{-\delta} y\right), \quad \delta=+,-, \quad y \rightarrow \infty,
$$

and separating points on $(K, \infty)$ :

$$
K<y_{1}<y_{2} \Rightarrow\left(E_{+}\left(y_{1}\right), E_{-}\left(y_{1}\right)\right) \neq\left(E_{+}\left(y_{2}\right), E_{-}\left(y_{2}\right)\right)
$$

The dense subset $\mathcal{D}$ is defined by (3.33)-(3.35) below. For expository simplicity, however, we shall summarize our results for a subset of $\mathcal{D}$, namely,

$$
\mathcal{D}_{\mathrm{irr}} \equiv\left\{\left(r, a_{+}, a_{-},\left(N_{+}+1\right) a_{+}-N_{-} a_{-}\right) \in \mathcal{E} \mid a_{+} / a_{-} \notin \mathrm{Q}, N_{+}, N_{-} \in \mathbb{N}\right\} .
$$

Since the $b$-values allowed here are dense in $\mathbb{R}$ for $a_{+} / a_{-}$irrational, $\mathcal{D}_{\text {irr }}$ is already dense in $\mathcal{E}$.

Fixing $\left(r, a_{+}, a_{-}, b\right) \in \mathcal{D}_{\text {irr }}$, any joint eigenfunction of $A_{+}$and $A_{-}$with eigenvalues $E_{+}(y)$ and $E_{-}(y)$, resp., is a linear combination of $\Psi(x, y)$ and $\Psi(-x, y)$. [More precisely, we prove that this holds true for all sufficiently large $y$; cf. Appendix B.] The latter are explicitly given by

$$
\begin{aligned}
\Psi(x, y)= & \mathcal{N} \prod_{j=-N_{+}}^{N_{+}} \frac{1}{s_{-}\left(x+i j a_{+}\right)} \cdot \prod_{\delta=+,-} \prod_{j=1}^{N_{\delta}} s_{-}\left(x+z_{j}^{\delta}(y)\right) \\
& \times \exp \left[i r x\left(2 N_{+} N_{-}+N_{+}+N_{-}+1\right)+i x y\right], \quad y \in(K, \infty) .
\end{aligned}
$$

The normalizing factor $\mathcal{N}$ depends on the parameters, but not on $x$ and $y$. The "zero functions" $z_{1}^{+}, \ldots, z_{N_{+}}^{+}$and $z_{1}^{-}, \ldots, z_{N_{-}}^{-}$are functions from $(K, \infty)$ to $i(0, \infty)$ that are real-analytic and such that

$$
\lim _{y \rightarrow \infty} z_{j}^{\delta}(y)=i j a_{\delta}, \quad j=1, \ldots, N_{\delta}, \quad \delta=+,-.
$$

These functions are determined as solutions to a certain constraint system. This system depends on the parameters in a quite complicated fashion, and for brevity we do not describe it here. [It is given by (3.5), (3.10), and (3.11); cf. also Appendix A.]

In view of $(1.23)$, the function $\Psi(x, y)$ has asymptotics

$$
\Psi(x, y) \sim c(x) \exp (i x y), \quad y \rightarrow \infty .
$$

Here, the $c$-function reads

$$
c\left(r, a_{+}, a_{-},\left(N_{+}+1\right) a_{+}-N_{-} a_{-} ; x\right)=\mathcal{N} \frac{\Pi_{k=1}^{N_{-}} s_{+}\left(x+i k a_{-}\right)}{\prod_{j=0}^{N_{+}} s_{-}\left(x-i j a_{+}\right)} \exp i r x\left(2 N_{+} N_{-}+N_{+}+N_{-}+1\right) .
$$

It is not obvious, but true, that the normalization constant $\mathcal{N}$ can be chosen such that 


$$
c\left(r, a_{+}, a_{-}, b ; x\right)=\frac{G\left(r, a_{+}, a_{-} ; x-i b+i\left(a_{+}+a_{-}\right) / 2\right)}{G\left(r, a_{+}, a_{-} ; x+i\left(a_{+}+a_{-}\right) / 2\right)},
$$

for parameters in $\mathcal{E}$ (1.14). Here, the function $G\left(r, a_{+}, a_{-} ; z\right)$ is the generalized elliptic gamma function from Ref. 14, which is meromorphic in $r, a_{+}, a_{-}$, and $z$ as long as $a_{+} r$ and $a_{-} r$ stay in the right half plane. The weight and scattering functions introduced in Ref. 14 can be written as

$$
\begin{gathered}
w(x)=\frac{1}{c(x) c(-x)} \\
u(x)=-\exp (-2 \operatorname{ir} x) \frac{c(x)}{c(-x)} .
\end{gathered}
$$

[This easily follows from their definition in terms of the $G$-function; to check (1.28) one also needs the defining A $\Delta$ Es of the latter; cf. Proposition III.8 in Ref. 14.] Thus, the joint eigenfunction

$$
\mathcal{F}(x, y)=w(x)^{1 / 2} \Psi(x, y)
$$

of the $\mathrm{A} \Delta \mathrm{Os} H_{+}$and $H_{-}$has plane wave asymptotics

$$
\mathcal{F}(x, y) \sim w(x)^{1 / 2} c(x) \exp (i x y)=[-\exp (2 i r x) u(x)]^{1 / 2} \exp (i x y), \quad y \rightarrow \infty .
$$

The obvious question arising from these results is now the following: Are there meromorphic joint eigenfunctions $\Psi(x, y)$ for arbitrary parameters in $\mathcal{E}(1.14)$ that depend continuously on the parameters and are proportional to $\Psi(x, y)(1.22)$ for parameters in $\mathcal{D}_{\text {irr }}$ ? (Since $\mathcal{D}_{\text {irr }}$ is dense in $\mathcal{E}$, such an interpolation is unique up to scale factors depending on $y$ and the parameters.) The point is that the same question has an affirmative answer for the $c$-function, as we have just seen.

The answer to the joint eigenfunction question, however, may well be "No." To see why, one need only note that when a sequence $\left(r, a_{+, n}, a_{-, n}, b_{n}\right) \in \mathcal{D}_{\text {irr }}$ converges to a point in $\mathcal{E}$, then the integers $N_{\delta, n}$ [cf. (1.21)] typically go to $\infty$. Thus, the poles of $\Psi(x, y)$ due to the first product in (1.22) become dense on the lines $\operatorname{Re} x=k \pi / r, k \in \mathbb{Z}$.

From this perspective our next result is quite surprising. To state it, we introduce the even function,

$$
\chi(x, y) \equiv \Psi(x, y)+\Psi(-x, y)
$$

Now consider a rectangle $|\operatorname{Re} x|<\pi / r,|\operatorname{Im} x|<L$. Fixing $a_{+}, a_{-}$, and a compact $b$-interval $I$, the number of poles of $\Psi(x, y)$ in the rectangle can be made arbitrarily large by choosing suitable $b \in I$; cf. the previous paragraph. By contrast, the number of poles of $\chi(x, y)$ in the rectangle is bounded above by a finite number that depends only on $L$ and $I$ !

To explain why this is true, we write

$$
\chi(x, y)=\mathcal{N} \prod_{j=-N_{+}}^{N_{+}} \frac{1}{s_{-}\left(x+i j a_{+}\right)}[\mathcal{H}(x, y)-\mathcal{H}(-x, y)]
$$

where $\mathcal{H}$ is the holomorphic function

$$
\mathcal{H}(x, y) \equiv \prod_{\delta=+,-} \prod_{j=1}^{N_{\delta}} s_{-\delta}\left(x+z_{j}^{\delta}(y)\right) \cdot \exp \left[\operatorname{irx}\left(2 N_{+} N_{-}+N_{+}+N_{-}+1\right)+i x y\right]
$$

The crux is now that one has the identities 


$$
\mathcal{H}\left(i k_{+} a_{+}+i k_{-} a_{-}, y\right)=\mathcal{H}\left(-i k_{+} a_{+}-i k_{-} a_{-}, y\right), \quad k_{\delta} \in\left\{-N_{\delta}, \ldots, 0, \ldots, N_{\delta}\right\}, \quad \delta=+,-
$$

Thus, poles of $\chi(x, y)$ on the imaginary axis can only occur for

$$
x=i k_{+} a_{+}+i k_{-} a_{-}, \quad k_{+} \in\left\{-N_{+}, \ldots, 0, \ldots, N_{+}\right\}, \quad\left|k_{-}\right|>N_{-},
$$

and so the assertion in the previous paragraph readily follows.

Now it is clear from (1.22) that we have

$$
\Psi(x \pm \pi / r, y)=\exp ( \pm i \pi y / r) \Psi(x, y) .
$$

Thus, $\Psi(x, y)$ is $\pi / r$-periodic or $\pi / r$-antiperiodic when $y / r$ is an integer. Defining

$$
\chi_{n}(x) \equiv \chi(x, n r), \quad n r>K, \quad n \in \mathbb{N},
$$

we therefore obtain a function that is $\pi / r$-periodic/antiperiodic for $n$ even/odd.

As a consequence, there is no apparent obstruction to the existence of a meromorphic interpolation for the functions $\chi_{n}(x)$. However, if an explicit representation for an interpolation exists, it is most likely vastly different from (1.22). Indeed, this is the case for the hyperbolic specialization, where we have found an interpolation in terms of a natural generalization of Euler's hypergeometric function; cf. Ref. 3 and papers to appear.

Before turning to orthogonality issues, we point out a consequence of the quasi-periodicity relations (1.36) that is of interest in itself, and that will be invoked later on. Recalling (1.3), we can rephrase (1.36) by saying that $\Psi(x, y)$ is an eigenfunction of the $\mathrm{A} \Delta \mathrm{Os} T_{ \pm \pi / r}$. Now this is true for $\Psi(-x, y)$, too, but then we obtain different eigenvalues. On the other hand, when we introduce the "quasi-periodicity $\mathrm{A} \Delta \mathrm{O}$, ,"

$$
Q \equiv T_{\pi / r}+T_{-\pi / r}
$$

then we obtain

$$
(Q \Psi)( \pm x, y)=E_{Q} \Psi( \pm x, y), \quad E_{Q} \equiv 2 \cos (\pi y / r) .
$$

Thus, as already mentioned above, the functions $\Psi( \pm x, y)$ are, in fact, joint eigenfunctions of three commuting independent $\mathrm{A} \Delta \mathrm{Os} A_{+}, A_{-}$, and $Q$.

Next, we describe results concerning orthogonality of the functions

$$
\Phi_{n}(x) \equiv w(x)^{1 / 2} \chi_{n}(x), \quad n r>K, \quad n \in \mathbb{N}, \quad x \in(0, \pi / r),
$$

in the Hilbert space $L^{2}((0, \pi / r), d x)$. The weight function $w(x)$ is given by

$$
w\left(r, a_{+}, a_{-},\left(N_{+}+1\right) a_{+}-N_{-} a_{-} ; x\right)=(-)^{N_{+}+N_{-}+1} \mathcal{N}^{-2} s_{-}(x)^{2} \frac{\Pi_{ \pm j=1}^{N_{+}} s_{-}\left(x-i j a_{+}\right)}{\Pi_{ \pm k=1}^{N_{-}} s_{+}\left(x-i k a_{-}\right)}
$$

cf. (1.25) and (1.27). It is non-negative for real $x$, and we take the positive square root in (1.40). Then the function $w(x)^{1 / 2}, x \in(0, \pi / r)$, has an analytic continuation to an odd, $\pi / r$-antiperiodic function that has no singularities for real $x$. The factor $s_{-}(x)$ in the latter function cancels the factor $1 / s_{-}(x)$ in (1.22), and the remaining poles do not meet the real axis (since $a_{+} / a_{-}$is irrational). Hence $\Phi_{n}(x)$ (1.40) extends to an odd function without singularities for real $x$, which is $\pi / r$-periodic/-antiperiodic for $n$ odd/even.

As a consequence, the functions $\Phi_{n}(x)$ are square-integrable on $(0, \pi / r)$. One of the principal results of this paper is now that these functions are pairwise orthogonal, provided the parameters belong to the region 


$$
\mathcal{C} \equiv\left\{\left(r, a_{+}, a_{-}, b\right) \in \mathcal{E} \mid 0<b<a_{+}+a_{-}\right\} .
$$

To provide more perspective on this parameter restriction, we mention that $\mathcal{C}$ coincides with the parameter region for which the $u$-function has winding number 0 as $x$ goes from 0 to $\pi / r$. Correspondingly, its logarithm has a rapidly convergent Fourier series. To be specific, we have [cf. Ref. 14 (4.87)]

$$
u\left(r, a_{+}, a_{-}, b ; x ; .\right)=\exp \left(2 i \sum_{j=1}^{\infty} \frac{\sinh \left(a_{+}-b\right) n r \sinh \left(a_{-}-b\right) n r}{n \sinh a_{+} n r \sinh a_{-} n r} \sin 2 n r x\right) .
$$

For convergence of the series one needs $|\operatorname{Im} x|<d / 2$, with

$$
d \equiv a_{+}+a_{-}-\left|a_{+}-b\right|-\left|a_{-}-b\right| .
$$

Thus, one gets $d>0$ iff the parameters belong to $\mathcal{C}(1.42)$. [Note that (1.43) exhibits symmetry under $a_{+} \leftrightarrow a_{-}$and $\left.b \rightarrow a_{+}+a_{-}-b.\right]$

Provided the parameters belong to $\mathcal{C}$, the $w$-function admits a similar representation. It is expedient to write, first of all,

$$
w\left(r, a_{+}, a_{-}, b ; x\right)=C^{2} s_{-}(x) s_{+}(x) w_{r}(x) .
$$

Here we have introduced the reduced weight function $w_{r}$, and the positive constant $C$ reads

$$
C\left(r, a_{+}, a_{-}\right)=2 r \prod_{k=1}^{\infty}\left[1-\exp \left(-2 k r a_{+}\right)\right]\left[a_{+} \rightarrow a_{-}\right]
$$

cf. Ref. 14 (5.41). Then one has, from Ref. 14 (5.54),

$$
w_{r}\left(r, a_{+}, a_{-}, b ; x\right)=\exp \left(\sum_{j=1}^{\infty} \frac{\sinh \left(a_{+}+a_{-}-2 b\right) n r}{n \sinh a_{+} n r \sinh a_{-} n r} \cos 2 n r x\right) .
$$

Just as for (1.43), the series converges in a strip containing the real axis iff the parameters belong to $\mathcal{C}$. (Note the strip width is larger than for the $u$-function when $a_{+}<b<a_{-}$, say. Note also that $w$ is symmetric under $a_{+} \leftrightarrow a_{-}$, but not under $b \rightarrow a_{+}+a_{-}-b$.)

Returning now to the Hilbert space $L^{2}((0, \pi / r), d x)$, we continue by pointing out that the restriction to $\mathcal{C}$ appears to be essential: For parameters outside $\mathcal{C}$, orthogonality is most likely violated, in general. Whenever this is the case, there exists no reinterpretation of the A $\Delta \mathrm{Os} H_{\delta}$ (1.12) as symmetric Hilbert space operators whose domains include the eigenfunctions $\Phi_{n}$ and whose action equals the obvious one.

By contrast, when we restrict the parameters to $\mathcal{C}$, then we obtain self-adjoint operators (denoted again $H_{+}, H_{-}$) on the closed subspace

$$
\mathcal{H}_{1}(K) \subset \mathcal{H}_{1} \equiv L^{2}((0, \pi / r), d x),
$$

spanned by the functions $\Phi_{n}, n>K / r$, by proceeding in the obvious way: We define

$$
H_{\delta} \Phi_{n} \equiv E_{\delta}(n r) \Phi_{n}, \quad n>K / r, \quad \delta=+,-,
$$

extend linearly, and then take the closure. Save for some special cases, we have not been able to prove our expectation that the orthocomplement of $\mathcal{H}_{1}(K)$ is spanned by joint eigenfunctions $\Phi_{n}, n=0,1, \ldots,[K / r]$, of the $\mathrm{A} \Delta \mathrm{Os} H_{\delta}$ with real eigenvalues $E_{\delta}(n r)$.

The functional-analytic problems involved in the above were discussed already in Refs. 2, 3; briefly, the Hilbert space theory of analytic difference operators (as opposed to discrete difference operators) is virtually nonexistent. Indeed, from the concrete examples we study: here and else- 
where it is likely that no straightforward generalization of the standard lore concerning selfadjointness and eigenfunction expansions for ordinary differential operators exists. Roughly speaking, our strategy is instead to exploit the properties of the explicit eigenfunctions to solve the orthogonality and self-adjointness problems simultaneously-with the above restrictions and provisos, however.

The results surveyed above are detailed in Secs. II-IV. Specifically, in Sec. II we restrict attention to the case $b=g a_{+}, g=2,3, \ldots$, which we already briefly considered at the end of our lecture notes. ${ }^{3}$ In this special case we need only appeal to the constraint system studied in Appendix A. This case has several other distinctive features compared to the general case. In particular, the "nonrelativistic limit" $a_{+} \downarrow 0$ can be handled, which gives rise to the integer $g$ eigenfunctions of the Lamé operator (1.1).

The general case studied in Sec. III is more involved. Roughly speaking, we wind up with two constraint systems of the type studied in Appendix A: one corresponding to $N_{+}$and the other to $N_{-}$; these two systems are coupled via the spectral variable $y$. In this way we can handle a dense subset $\mathcal{D}$ of $\mathcal{E}(1.14)$ [which contains (1.21)], but our knowledge about the analytic properties of the eigenfunctions neither suffices to deduce the existence of an interpolation (as discussed above) nor enables us to say anything about the eigenfunctions of the Lamé operator for $g$ not equal to an integer. [Observe that the latter can be formally obtained already via sequences in $D_{\mathrm{irr}}(1.21)$. Since $N_{+}$and/or $N_{-}$must go to $\propto$ in this limit, we are losing control of the eigenfunction limit, however.]

Section IV is mainly devoted to a study of self-adjointness and orthogonality questions. The principal results have already been summarized above. Here we add that we find it convenient to perform a second similarity transformation to $\mathrm{A} \Delta \mathrm{Os} B_{+}$and $B_{-}$whose structure is quite close to

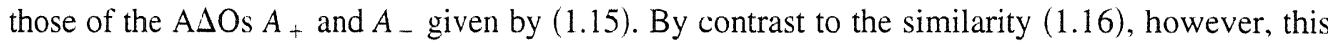
second similarity does not admit an interpolation to all of the elliptic parameter domain $\mathcal{E}(1.14)$. Even so, $A_{\delta}$ and $B_{\delta}$ are sufficiently close to enable us to enlarge the parameter set for which joint $\left(A_{+}, A_{-}\right)$-eigenfunctions [and hence $\left(H_{+}, H_{-}\right)$-eigenfunctions] can be found. The pertinent enlargement is somewhat involved; furthermore, the relation between $A_{\delta}$ and $B_{\delta}$ may be quite confusing on the first acquaintance. The last part of Sec. IV, where we detail the extension, can be more easily understood for the hyperbolic specialization; cf. Ref. 11.

In Appendix A we handle the constraint system associated with the zern representation when one of the integers $N_{+}, N_{-}$vanishes. More precisely, we study a more general system that exhibits most (but not all) of the relevant features of the former system.

In Appendix B we first collect some results on second order analytic difference equations, associated with the notion of a Casorati determinant. These well-known results are used to prove Theorem B.1. Roughly speaking, this theorem says that the meromorphic functions $\Psi( \pm x, y)$ are a basis for the joint $A_{\delta}$-eigenspace, provided the quotient $a_{+} / a_{-}$is irrational and the spectral variable $y$ is large enough. [In fact, we work with the similarity transforms $B_{\delta}$ and their holomorphic eigenfunctions $\mathcal{H}( \pm x, y)$.]

\section{EIGENFUNCTIONS FOR THE INTEGER-g CASE}

In this section we choose

$$
g=N+1=2,3, \ldots,
$$

in the $\mathrm{A} \Delta \mathrm{O} H_{\text {rel }}$ (1.2), and accordingly obtain eigenfunctions reducing to the Lamé functions for $\beta \rightarrow 0$. [Pushing the shifts to the right in (1.2), one sees that the $g=1$ case is trivial, just as for $H_{\mathrm{nr}}$ (1.1).] To ease the notation in this section, it is convenient to trade the parameters $a_{+}, a_{-}$, and $b$ in the A $\Delta$ Os (1.12)-(1.16) for

$$
a_{+}=-i v, \quad a_{-}=a, \quad b=-i(N+1) v,
$$

and to work with suitable positive multiples of the $\mathrm{A} \Delta \mathrm{Os}$ (1.15). 
Specifically, we start from the $A_{\text {_-multiple }}$

$$
A \equiv \frac{s(x-(N+1) v)}{s(x)} T_{v}+(v \rightarrow-v), \quad s(x) \equiv s(r, a ; x),
$$

and at first restrict $v$ by requiring

$$
2 N v \in i(0, a) .
$$

Substituting (2.2) in (1.22), we obtain (note $N_{-}=0$ )

$$
\Psi(x, y)=\mathcal{N} \prod_{j=-N}^{N} \frac{1}{s(x+j v)} \cdot \prod_{j=1}^{N} s\left(x+z_{j}(y)\right) \cdot \exp (x \Sigma),
$$

with

$$
\Sigma \equiv i r(N+1)+i y .
$$

In the hyperbolic case $(r=0)$ the existence of eigenfunctions of this form can be deduced for arbitrary $y$. This is because a second far more explicit form of the eigenfunctions exists in that case (cf. Ref. 2 and Sec. II in Ref. 11), from which the existence of the factorized representation (2.5) is readily deduced.

Staying with the elliptic case, one may view (2.5) as an Ansatz for the eigenvalue equation $A \Psi=E \Psi$. Doing so, one readily verifies the identity

$$
\Psi(x, y)^{-1}(A \Psi)(x, y)=E\left(\Sigma, z_{1}, \ldots, z_{N} ; x\right),
$$

where

$$
E \equiv \frac{1}{s(x)}\left(s(x+N v) e^{-v \Sigma} \prod_{j=1}^{N} \frac{s\left(x-v+z_{j}\right)}{s\left(x+z_{j}\right)}+(v \rightarrow-v)\right) .
$$

Obviously, the function $E$ is elliptic in $x$ with periods $\pi / r, i a$, independently of the choice of $\Sigma, z_{1}, \ldots, z_{N} \in \mathrm{C}$. Choosing from now on the numbers $z_{1}, \ldots, z_{N}$ pairwise incongruent and incongruent to 0 (modulo the period lattice), the two summands of $E$ have simple poles at $x \equiv 0$, $-z_{1}, \ldots,-z_{N}$.

As a consequence, we obtain an eigenfunction whenever the residues at all of these poles cancel. (Indeed, this entails that $E$ is constant.) Now for $x=0$ we need

$$
e^{-v \Sigma} \prod_{j=1}^{N} s\left(-v+z_{j}\right)-(v \rightarrow-v)=0,
$$

so that we must have

$$
\Sigma=\frac{1}{2 v} \ln \left(\prod_{j=1}^{N} \frac{s\left(z_{j}-v\right)}{s\left(z_{j}+v\right)}\right)
$$

Substituting this in (2.8), we now study whether the residues at $x=-z_{k}$ can be made to cancel. For this we clearly need

$$
s\left(z_{k}-N v\right) \prod_{\substack{j=1 \\ j \neq k}}^{N} s\left(z_{j}-z_{k}-v\right) \prod_{j=1}^{N} s\left(z_{j}+v\right)-(v \rightarrow-v)=0,
$$

where $k=1, \ldots, N$. 
The system (2.11) of $N$ equations for $N$ unknowns $z_{1}, \ldots, z_{N}$ is a special case of the constraint system mentioned in Sec. I. Introducing the function

$$
f(w)=s(v w) / v, \quad s(z) \equiv s(r, a ; z),
$$

it can be rewritten as a concrete form of the system (A2)-(A3) studied in Appendix A. Thus we obtain solutions

$$
z_{1}=v+t v, \quad z_{l}=l v+O\left(t^{2}\right), \quad d z_{l} / d t=O(t), \quad l=2, \ldots, N, \quad t \rightarrow 0,
$$

to the equations (2.11) with $k=2, \ldots, N$.

Now for a general function $f(w)$ it would not follow that the solution (A8) to the system (A7) also solves the larger system (A3). Due to the ancestry of the special case (2.12) of the system, however, we may deduce that one also has $F_{1}(W(t))=0$. Indeed, inserting (2.13) and (2.10) in the elliptic function $E\left(\Sigma, z_{1}, \ldots, z_{N} ; x\right)$, we obtain vanishing residues at $x \equiv 0,-z_{2}, \ldots,-z_{N}$, so the residues at $x \equiv-z_{1}$ must vanish, too. (Recall that a nonconstant elliptic function must have more than one pole in a period cell.)

Consequently, the system (2.11) with $k=1, \ldots, N$ admits a holomorphic solution curve $z_{1}(t), \ldots, z_{N}(t)$ of the form (2.13) for $t$ near 0. Moreover, we may and will choose $\epsilon>0$ small enough so that we have

$$
z_{j}(t) \in i(0, \infty), \quad s\left(z_{j}(t) \pm v\right) \neq 0, \quad s\left(z_{j}(t)+N v\right) \neq 0, \quad j=1, \ldots, N,
$$

for all $t \in(0, \epsilon)$. This ensures that

$$
y(t)=-(N+1) r-\frac{i}{2 v} \ln \left(\prod_{j=1}^{N} \frac{s\left(z_{j}(t)-v\right)}{s\left(z_{j}(t)+v\right)}\right)
$$

is a real-valued, real-analytic function on $(0, \epsilon)$. Moreover, since the function $E(2.8)$ is $x$-independent, we may choose $x=N v$, yielding

$$
E=\mu \exp (-i r v(N+1)-i y v), \quad \mu \equiv \frac{s(2 N v)}{s(N v)} \prod_{j=1}^{N} \frac{s\left((N-1) v+z_{j}\right)}{s\left(N v+z_{j}\right)} .
$$

Clearly, $\mu(t)$ is holomorphic at $t=0$ and satisfies

$$
\mu(t)=1+t v\left(\frac{s^{\prime}(N v)}{s(N v)}-\frac{s^{\prime}((N+1) v)}{s((N+1) v)}\right)+O\left(t^{2}\right), \quad t \rightarrow 0 .
$$

Next, we observe that $y^{\prime}(t)$ is analytic in a neighborhood of $t=0$ but for a simple pole at the origin [cf. (2.15)]:

$$
y^{\prime}(t)=-\frac{i}{2 v t}+O(1), \quad t \rightarrow 0
$$

Eventually decreasing $\epsilon$, we can therefore ensure

$$
y^{\prime}(t)<0, \quad t \in(0, \epsilon) .
$$

Then $y$ decreases monotonically from $\infty$ to $L_{\epsilon}-N-1 \equiv K$ as $t$ goes from 0 to $\epsilon$. Thus we may and will trade the parameter $t$ for $y$.

To proceed, we observe that we have

$$
\frac{d}{d y} \mu(t(y))=\mu^{\prime}(t)\left(y^{\prime}(t)\right)^{-1} \rightarrow 0, \quad y \rightarrow \infty
$$


cf. (2.17) and (2.18). In view of (2.16), this entails

$$
\frac{d}{d y} E \sim-i v \exp (-i r v(N+1)-i y v), \quad y \rightarrow \infty .
$$

(Recall that $f \sim g$ stands for $f / g \rightarrow 1$.) To obtain the asymptotics of $E=E(y)$, we note that $(2.15)$ yields

$$
y(t)=-(N+1) r-\frac{i}{2 v} \ln \left(\frac{t v s(v)}{s(N v) s((N+1) v)}\right)+O(t), \quad t \rightarrow 0 .
$$

Combining this with (2.16) and (2.17), we readily deduce

$$
E=\exp (-i v r(N+1)-i v y)+c_{N} \exp (i v r(N+1)+i v y)+O(\exp (3 i v y)), \quad y \rightarrow \infty,
$$

with

$$
c_{N} \equiv\left[s^{\prime}(N v) s((N+1) v)-s^{\prime}((N+1) v) s(N v)\right] / s(v) .
$$

The results obtained thus far hold true when $v$ satisfies (2.4). Indeed, this restriction guarantees first of all that the hypothesis (A6) in Theorem A.1 is satisfied; cf. (2.12). But it also enables us to ensure a well-defined eigenvalue formula (2.16).

Let us now require, more generally,

$$
v \in i(0, \infty), \quad k v \notin i \mathrm{~N} a, \quad k=1, \ldots, 2 N .
$$

Then we arrive at the same results as before, but for a subtle change: To guarantee the reality of $y$ for $t$ near 0 we may have to choose $t$ in an interval $(-\epsilon, 0)$. This eventual sign change depends on the sign of the product in (2.15) for $t$ near 0 . In view of (2.13) this sign equals the sign of $v s(v) / s(N v) s((N+1) v)$; cf. also the asymptotics (2.22).

Next, we introduce the $\mathrm{A} \Delta \mathrm{O}$

$$
\widetilde{A} \equiv(-)^{N+1} e^{2 i(N+1) r x} T_{i a}+(i \rightarrow-i) .
$$

Using the iterated $\mathrm{A} \Delta \mathrm{E}(1.10)$, one infers that $\tilde{A}$ equals a positive multiple of the $\mathrm{A} \Delta \mathrm{O} A_{+}(1.15)$; cf. (2.2). It is readily verified that $\Psi(x, y)(2.5)$ is an eigenfunction of $\tilde{A}$, with the eigenvalue

$$
\widetilde{E}=\exp \left(2 i r \sum_{j=1}^{N} z_{j}(y)\right) e^{a y}+\exp \left(-2 i r \sum_{j=1}^{N} z_{j}(y)\right) e^{-2(N+1) a r} e^{-a y} .
$$

(Notice that this actually holds true for an arbitrary dependence of $z_{j}$ on $y$. ) Clearly, we have

$$
\widetilde{E} \sim \exp (i N(N+1) r v) e^{a y}, \quad d \widetilde{E} / d y \sim a \exp (i N(N+1) r v) e^{a y}, \quad y \rightarrow \infty .
$$

In summary, we have arrived at joint eigenfunctions,

$$
\Psi(x, y)=\mathcal{N} \prod_{j=-N}^{N} \frac{1}{s(x+j v)} \cdot \prod_{j=1}^{N} s\left(x+z_{j}\right) \cdot \exp \left(\frac{x}{2 v} \ln \left(\prod_{j=1}^{N} \frac{s\left(z_{j}-v\right)}{s\left(z_{j}+v\right)}\right)\right),
$$

of the two A $\Delta$ Os $A(2.3)$ and $\widetilde{A}$ (2.26), with eigenvalues $E$ (2.16) and $\widetilde{E}$ (2.27), respectively, and with $y \in(K, \infty)$ and parameters restricted solely by $(2.25)$. The functions $z_{1}, \ldots, z_{N}$ are solutions to the constraint system (2.11) of the form (2.13), and the solution curve parameters $t$ and $y$ are 
related via (2.15). Since the $y$-derivatives of both eigenvalues are positive for $y$ large, an eventual increase of $K$ ensures that the eigenvalue pair $(E(y), \widetilde{E}(y))$ separates points on $(K, \infty)$.

We proceed by obtaining the "nonrelativistic limit" $v \rightarrow 0$ of the constraints (2.10), (2.11) and eigenfunctions (2.29). First, (2.10) gives rise to

$$
\Sigma=-\sum_{j=1}^{N} \frac{s^{\prime}\left(z_{j}\right)}{s\left(z_{j}\right)}
$$

Second, dividing (2.11) by $v$ and taking $v$ to 0 yields

$$
N \frac{s^{\prime}\left(z_{k}\right)}{s\left(z_{k}\right)}+\sum_{\substack{j=1 \\ j \neq k}}^{N} \frac{s^{\prime}\left(z_{j}-z_{k}\right)}{s\left(z_{j}-z_{k}\right)}-\sum_{j=1}^{N} \frac{s^{\prime}\left(z_{j}\right)}{s\left(z_{j}\right)}=0, \quad k=1, \ldots, N .
$$

Proceeding formally, we can also take $v$ to 0 in the eigenfunction (2.29), yielding the limit function

$$
\Psi_{0}(x, y)=\mathcal{N}_{0} s(x)^{-2 N-1} \prod_{j=1}^{N} s\left(x+z_{j}\right) \exp \left[-x s^{\prime}\left(z_{j}\right) / s\left(z_{j}\right)\right], \quad y=-(N+1) r+i \sum_{j=1}^{N} \frac{s^{\prime}\left(z_{j}\right)}{s\left(z_{j}\right)} .
$$

Now (1.5) entails

$$
s^{\prime}(x) / s(x)=\zeta(x)-2 \eta x r / \pi,
$$

where $\zeta$ is the Weierstrass $\zeta$-function. Therefore, the functions (2.32) and constraints (2.30), (2.31) amount to the Lamé functions, and associated constraints, as specified by Whittaker and Watson; cf. p. 572 and p. 574, respectively, of Ref. 4. We do not have sufficient information on the solution curve to rigorously control the above limits, though.

Next, we derive a crucial property of the holomorphic function

$$
\mathcal{H}_{N}(x) \equiv \prod_{j=1}^{N} s\left(x+z_{j}\right) \cdot \exp \left(\frac{x}{2 v} \ln \left(\prod_{j=1}^{N} \frac{s\left(z_{j}-v\right)}{s\left(z_{j}+v\right)}\right)\right)
$$

with $z_{1}, \ldots, z_{N}$ the above solutions to the constraint system (2.11). We have suppressed the $y$-dependence to prevent ambiguities in the next section. We can do so, since the property holds for arbitrary $y$; It reads

$$
\mathcal{H}_{N}(k v)=\mathcal{H}_{N}(-k v), \quad k=1, \ldots, N
$$

(As will become clear later on, this algebraic property is a key ingredient in our orthogonality analysis.)

The only $k$-value for which (2.35) is immediate from (2.34) is $k=1$. Indeed, in that case it holds true for $z_{1}, \ldots, z_{N}$ having arbitrary $y$-dependence. In order to prove (2.35), we exploit the $\mathrm{A} \Delta \mathrm{E}$

$$
B \mathcal{H}_{N}=E \mathcal{H}_{N}, \quad B \equiv\left(\prod_{j=-N}^{N} s(x+j v)\right) A\left(\prod_{j=-N}^{N} s(x+j v)\right)^{-1},
$$

satisfied by $\mathcal{H}_{N} ;$ cf. (2.29). Recalling (2.3), we obtain

$$
B=\frac{s(x+N v)}{s(x)} T_{v}+(v \rightarrow-v)
$$


so this $\mathrm{A} \Delta \mathrm{E}$ can be written as

$$
s(x+N v) \mathcal{H}_{N}(x-v)+s(x-N v) \mathcal{H}_{N}(x+v)=E s(x) \mathcal{H}_{N}(x) .
$$

First, we put $x=0$ in (2.38). Using the oddness of $s(x)$ and the restriction (2.25), this yields $\mathcal{H}_{N}(-v)=\mathcal{H}_{N}(v)$. Now we proceed recursively. Assuming $(2.35)$ for $k=1, \ldots, l$ with $l<N$, we first substitute $x=l v$ in (2.38) to obtain

$$
s((l+N) v) \mathcal{H}_{N}((l-1) v)+s((l-N) v) \mathcal{H}_{N}((l+1) v)=E s(l v) \mathcal{H}_{N}(l v) .
$$

Next, we put $x=-l v$ and use the assumption and the oddness of $s(x)$ to get

$$
s((l-N) v) \mathcal{H}_{N}(-(l+1) v)+s((l+N) v) \mathcal{H}_{N}((l-1) v)=E s(l v) \mathcal{H}_{N}(l v)
$$

Comparing (2.39) and (2.40), we obtain $\mathcal{H}_{N}(k v)=\mathcal{H}_{N}(-k v)$ for $k=l+1$, since $s((l-N) v)$ $\neq 0$; cf. (2.25). Thus, the asserted identities (2.35) readily follow.

Besides their use in the orthogonality problem, the identities (2.35) have two further striking consequences. First, consider the function (Casorati determinant)

$$
C_{N}(x) \equiv \mathcal{H}_{N}(x+v / 2) \mathcal{H}_{N}(-x+v / 2)-\mathcal{H}_{N}(x-v / 2) \mathcal{H}_{N}(-x-v / 2)
$$

Due to (2.35), it satisfies

$$
C_{N}(n v)=0, \quad n=-N+1 / 2,-N+3 / 2, \ldots, N-1 / 2 .
$$

This is easily seen to entail the remarkably simple result

$$
C_{N}(x)=\alpha_{N} \prod_{n=-N+1 / 2}^{N-1 / 2} s(x-n v)
$$

where $\alpha_{N}$ does not depend on $x$. [Indeed, the quotient of $C_{N}(x)$ and the product on the rhs is elliptic with periods $\pi / r, i a$, and pole-free in view of (2.42).]

Second, combining (2.35) and (2.34), one deduces

$$
\prod_{j=1}^{N} \frac{s\left(z_{j}-k v\right)}{s\left(z_{j}+k v\right)}=\prod_{j=1}^{N}\left(\frac{s\left(z_{j}-v\right)}{s\left(z_{j}+v\right)}\right)^{k}, \quad k=2, \ldots, N .
$$

Thus, the asymptotics (2.13) can be rendered far more precise. Indeed, from (2.13) and (2.44) one readily obtains

$$
z_{l}=l v+d_{l} v t^{l}+O\left(t^{l+2}\right), \quad l=2, \ldots, N, \quad t \rightarrow 0,
$$

where

$$
d_{l} \equiv v^{l-1} \prod_{j=1}^{N} \frac{s(j v+l v)}{s(j v+v)^{l}} \cdot \prod_{j=2}^{N} s(j v-v)^{l} \cdot \prod_{\substack{j=l \\ j \neq 1}}^{N} \frac{1}{s(j v-l v)}, \quad l=2, \ldots, N
$$

Note that these coefficients are real and nonzero due to (2.25). Moreover, one has

$$
\lim _{v \rightarrow 0} d_{l}=\prod_{j=1}^{N} \frac{j+l}{(j+1)^{l}} \cdot \prod_{j=2}^{N}(j-1)^{l} \cdot \prod_{\substack{j=1 \\ j \neq l}}^{N} \frac{1}{(j-l)}, \quad l=2, \ldots, N .
$$




\section{EIGENFUNCTIONS FOR A DENSE PARAMETER SET}

In this section we take the $\mathrm{A} \Delta \mathrm{Os} A_{+}, A_{-}$(1.15) with

$$
b=\left(N_{+}+1\right) a_{+}-N_{-} a_{-}, \quad N_{+}, N_{-} \in \mathbb{N}^{*},
$$

as our starting point. Using the $\mathrm{A} \Delta \mathrm{E}$ (1.10), they can be rewritten as

$$
\begin{aligned}
A_{+}= & (-)^{N_{+}+1} \exp \left[a_{+} r\left(N_{+}+1\right) N_{+}-a_{-} r\left(2 N_{+}+1\right) N_{-}\right] \\
& \times\left(e^{2 i r\left(N_{+}+1\right) x} \frac{s_{+}\left(x+i N_{-} a_{-}\right)}{s_{+}(x)} T_{i a_{-}}+(i \rightarrow-i)\right), \\
A_{-}= & (-)^{N_{-}} \exp \left[a_{-} r\left(N_{-}+1\right) N_{-}-a_{+} r\left(2 N_{-}+1\right)\left(N_{+}+1\right)\right] \\
& \times\left(e^{-2 i r N_{-} x} \frac{s_{-}\left(x-i\left(N_{+}+1\right) a_{+}\right)}{s_{-}(x)} T_{i a_{+}}+(i \rightarrow-i)\right) .
\end{aligned}
$$

In the hyperbolic case, the existence of joint eigenfunctions of the form

$$
\Psi(x, y)=\mathcal{N} \prod_{j=-N_{+}}^{N_{+}} \frac{1}{s_{-}\left(x+i j a_{+}\right)} \cdot \prod_{j=1}^{N_{+}} s_{-}\left(x+z_{j}^{+}(y)\right) \cdot \prod_{j=1}^{N_{-}} s_{+}\left(x+z_{j}^{-}(y)\right) \cdot e^{x \Sigma},
$$

with

$$
\Sigma \equiv i r\left(2 N_{+} N_{-}+N_{+}+N_{-}+1\right)+i y,
$$

can be deduced for arbitrary $y$; cf. Sec. III in Ref. 11. In the elliptic case we view (3.4) as an Ansatz for solving the $\mathrm{A} \Delta \mathrm{Es}$

$$
A_{\delta} \Psi=E_{\delta} \Psi, \quad \delta=+,-
$$

Correspondingly, we calculate the functions

$$
\Psi(x, y)^{-1}\left(A_{\delta} \Psi\right)(x, y)=E_{\delta}\left(\Sigma, z^{+}, z^{-} ; x\right), \quad \delta=+,-.
$$

Using (1.6), this readily yields

$$
\begin{aligned}
E_{\delta}= & e_{\delta} \frac{1}{s_{\delta}(x)}\left(\exp \left(2 i r \sum_{j=1}^{N_{\delta}} z_{j}^{\delta}\right) s_{\delta}\left(x+i N_{-\delta} a_{-\delta}\right) \exp \left(-i a_{-\delta} \Sigma\right)\right. \\
& \left.\times \prod_{j=1}^{N_{-} \delta} \frac{s_{\delta}\left(x-i a_{-\delta}+z_{j}^{-\delta}\right)}{s_{\delta}\left(x+z_{j}^{-\delta}\right)}+(i \rightarrow-i)\right), \quad \delta=+,-,
\end{aligned}
$$

with

$$
e_{\delta} \equiv \exp \left[a_{\delta} r\left(N_{\delta}+1\right) N_{\delta}-a_{-\delta} r\left(2 N_{+} N_{-}+N_{+}+N_{-}+1\right)\right] .
$$

Clearly, $E_{\delta}$ is elliptic in $x$ with periods $\pi / r, i a_{\delta}$. From now on we choose the numbers $z_{1}^{-\delta}, \ldots, z_{N-\delta}^{-\delta}$ pairwise incongruent and incongruent to 0 modulo the period lattice $\pi r^{-1} \mathrm{Z}$ $+i a_{\delta} Z$, so that the summands have only simple poles.

It is expedient to study first one of these two elliptic functions. To minimize signs, we concentrate on the function $E_{-\delta}$ and study if and when the residues at all of its poles can be made to vanish, so as to obtain an $A_{-\delta}$-eigenfunction with eigenvalue $E_{-\delta}$. For $x=0$ it suffices to require 


$$
\Sigma=\frac{2 r}{a_{\delta}} \sum_{j=1}^{N_{-} \delta} z_{j}^{-\delta}+\frac{1}{2 i a_{\delta}} \ln \left(\prod_{j=1}^{N_{\delta}} \frac{s_{-\delta}\left(z_{j}^{\delta}-i a_{\delta}\right)}{s_{-\delta}\left(z_{j}^{\delta}+i a_{\delta}\right)}\right)
$$

Substituting this in $E_{-\delta}$, we require next

$$
s_{-\delta}\left(z_{k}^{\delta}-i N_{\delta} a_{\delta}\right) \prod_{\substack{j=1 \\ j \neq k}}^{N_{\delta}} s_{-\delta}\left(z_{j}^{\delta}-z_{k}^{\delta}-i a_{\delta}\right) \prod_{j=1}^{N_{\delta}} s_{-\delta}\left(z_{j}^{\delta}+i a_{\delta}\right)-(i \rightarrow-i)=0,
$$

where $k=1, \ldots, N_{-}$. Whenever these requirements are met, we obtain vanishing residues at all poles. Accordingly, the elliptic function $E_{-\delta}$ reduces to a constant, and so we obtain an $A_{-} \delta$-eigenfunction.

Introducing the function

$$
f(w)=s_{-\delta}\left(i a_{\delta} w\right) / i a_{\delta},
$$

the constraint system (3.11) turns into a special case of the system studied in Appendix A. Now this special case arose already in the previous section. Requiring henceforth

$$
k a_{\delta} \notin \mathbb{N} a_{-\delta}, \quad k=1, \ldots, 2 N_{\delta},
$$

we can therefore deduce the existence of solutions $z_{j}^{\delta}\left(t_{\delta}\right)$ to (3.11) that are holomorphic at $t=0$ and satisfy

$$
z_{1}^{\delta}=i a_{\delta}\left(1+t_{\delta}\right), \quad z_{l}^{\delta}=i a_{\delta}\left(l+d_{\delta,} t_{\delta}^{l}\right)+O\left(t_{\delta}^{l+2}\right), \quad l=2, \ldots, N_{\delta}, \quad t_{\delta} \rightarrow 0,
$$

where

$$
d_{\delta, l}=\left(i a_{\delta}\right)^{l-1} \prod_{j=1}^{N_{\delta}} \frac{s_{-\delta}\left(i(j+l) a_{\delta}\right)}{s_{-\delta}\left(i(j+1) a_{\delta}\right)^{l}} \cdot \prod_{j=2}^{N_{\delta}} s_{-\delta}\left(i(j-1) a_{\delta}\right)^{l} \cdot \prod_{\substack{j=1 \\ j \neq l}}^{N_{\delta}} \frac{1}{s_{-\delta}\left(i(j-l) a_{\delta}\right)}
$$

Substituting these solutions, we deduce as before that $E_{-\delta}$ does not depend on $x$. Taking, for example, $\delta=+$ in (3.10)-(3.14), we therefore obtain an $A_{-}$-eigenfunction with eigenvalue $E_{-}$, independently of the choice of $z_{1}^{-}, \ldots, z_{N_{-}}$. But in order to obtain a joint eigenfunction of $A_{+}$and $A_{-}$, the requirements (3.10)-(3.11) must be met simultaneously for $\delta=+$ and $\delta=-$.

This can be achieved as follows. Consider the functions

$$
g_{\delta}\left(t_{\delta}\right) \equiv \frac{2 i r}{a_{-\delta}} \sum_{j=1}^{N_{\delta}} z_{j}^{\delta}-\frac{1}{2 a_{\delta}} \ln \left(\prod_{j=1}^{N_{\delta}} \frac{s_{-\delta}\left(z_{j}^{\delta}-i a_{\delta}\right)}{s_{-\delta}\left(z_{j}^{\delta}+i a_{\delta}\right)}\right), \quad \delta=+,-,
$$

where $z^{\delta}=z^{\delta}\left(t_{\delta}\right)$ is the above solution to (3.11). Letting $t_{\delta}$ vary over $\left(-\epsilon_{\delta}, 0\right)$ or $\left(0, \epsilon_{\delta}\right)$ (the choice being determined by positivity of the product), the functions $g_{\delta}: t_{\delta} \mapsto u$ are real-valued, real-analytic, and monotone for $\epsilon_{\delta}$ small enough, and $u$ goes to $\infty$ for $t_{\delta} \rightarrow 0$. Thus the inverse functions $h_{\delta}: u \mapsto t_{\delta}$ are well defined for $u$ varying over an interval $I_{\delta}=\left(\rho_{\delta}, \infty\right)$, and they are real-analytic and monotone on $I_{\delta}$.

Letting now $\rho=\max \left(\rho_{+}, \rho_{-}\right)$, we may view $t_{\delta}$ as a function $h_{\delta}(u)$ on $(\rho, \infty)$. Doing so, we define [cf. (3.5) and (3.10)]

$$
\begin{aligned}
y(u) & \equiv-r\left(2 N_{+} N_{-}+N_{+}+N_{-}+1\right)-\frac{2 i r}{a_{\delta}} \sum_{j=1}^{N_{-} \delta} z_{j}^{-\delta}-\frac{1}{2 a_{\delta}} \ln \left(\prod_{j=1}^{N_{\delta}} \frac{s_{-\delta}\left(z_{j}^{\delta}-i a_{\delta}\right)}{s_{-}\left(z_{j}^{\delta}+i a_{\delta}\right)}\right) \\
& =-r\left(2 N_{+} N_{-}+N_{+}+N_{-}+1\right)-2 i r \sum_{\delta=+,-} \frac{1}{a_{\delta}} \sum_{j=1}^{N_{-} \delta} z_{j}^{-\delta}+u,
\end{aligned}
$$


where $z^{\delta}=z^{\delta}\left(h_{\delta}(u)\right)$ and $u \in(\rho, \infty)$. Eventually increasing $\rho$, we deduce from (3.14) and holomorphy in $t_{\delta}$ that $y(u)$ is a real-analytic, increasing function on $(\rho, \infty)$, taking values in some interval $(K, \infty)$. Thus, we may and will view $u$ as a real-analytic function of $y$ on $(K, \infty)$.

The upshot is that there exist real-analytic functions

$$
(K, \infty) \rightarrow i(0, \infty), \quad y \mapsto z_{l}^{\delta}\left(h_{\delta}(u(y))\right), \quad l=1, \ldots, N_{\delta}, \quad \delta=+,-
$$

[denoted once more by $z_{l}^{\delta}(y)$ ], such that (3.10) and (3.11) with $k=1, \ldots, N_{-\delta}$ are satisfied both for $\delta=+$ and for $\delta=-$. As a result, we obtain a joint eigenfunction $\Psi(x, y), y \in(K, \infty)$, of the A $\Delta$ Os $A_{+}$and $A_{-}$, as advertised. Eventually increasing $K$, we can ensure

$$
s_{\delta}\left(i N_{-\delta} a_{-\delta}+z_{j}^{-\delta}(y)\right) \neq 0, \quad j=1, \ldots, N_{\delta}, \quad \delta=+,-,
$$

for all $y \in(K, \infty)$. Then we choose $x=i N_{-\delta} a_{-\delta}$ in (3.8), yielding

$$
\begin{aligned}
E_{\delta}(y)= & \exp \left[a_{\delta} r\left(N_{\delta}+1\right) N_{\delta}+2 i r \sum_{j=1}^{N_{\delta}} z_{j}^{\delta}(y)+a_{-\delta} y\right] \\
& \times \frac{s\left(2 i N_{-\delta} a_{-\delta}\right)}{s\left(i N_{-} a_{-\delta}\right)} \prod_{j=1}^{N_{-}} \frac{s_{\delta}\left(i\left(N_{-\delta}-1\right) a_{-\delta}+z_{j}^{-\delta}(y)\right)}{s_{\delta}\left(i N_{-\delta} a_{-\delta}+z_{j}^{-\delta}(y)\right)}, \quad \delta=+,-
\end{aligned}
$$

Clearly, these functions are real-valued and real-analytic on $(K, \infty)$.

The $y \rightarrow \infty$ asymptotics of $E_{\delta}$ is readily determined from the above. First, let us note that (3.5) and (3.10) entail

$$
\left|t_{\delta}\right|=O\left(\exp \left(-2 a_{\delta} y\right)\right), \quad y \rightarrow \infty .
$$

Now from (3.14), we deduce

$$
\begin{gathered}
E_{\delta}=\exp \left(a_{-\delta} y\right)\left(1-2 a_{\delta} r t_{\delta}+i a_{-\delta} d_{-\delta} t_{-\delta}+O\left(t_{+}^{2}\right)+O\left(t_{-}^{2}\right)\right), \quad y \rightarrow \infty, \\
d_{-\delta} \equiv \frac{s_{\delta}^{\prime}\left(i N_{-\delta} a_{-\delta}\right)}{s_{\delta}\left(i N_{-\delta} a_{-\delta}\right)}-\frac{s_{\delta}^{\prime}\left(i\left(N_{-\delta}+1\right) a_{-\delta}\right)}{s_{\delta}\left(i\left(N_{-\delta}+1\right) a_{-\delta}\right)} .
\end{gathered}
$$

Thus, we conclude

$$
E_{\delta}(y)=\exp \left(a_{-\delta} y\right)\left(1+O\left(\exp \left[-2 \min \left(a_{+}, a_{-}\right) y\right]\right)\right), \quad y \rightarrow \infty .
$$

Moreover, one readily verifies that

$$
\frac{d}{d y} z_{l}^{\delta}(y) \rightarrow 0, \quad l=1, \ldots, N_{\delta}, \quad \delta=+,-, \quad y \rightarrow \infty,
$$

so that (3.20) entails

$$
\frac{d}{d y} E_{\delta}(y) \sim a_{-\delta} \exp \left(a_{-\delta} y\right), \quad y \rightarrow \infty
$$

From this large- $y$ asymptotics we see that $E_{\delta}(y)$ is an increasing function of $y$ for $y$ sufficiently large. Thus, eventually increasing $K$, we may and will assume that the eigenvalue pair separates points on $(K, \infty)$. [I.e., $(1.20)$ holds true.]

Summarizing, we have proved the existence of joint eigenfunctions when the parameter $b$ is given by (3.1) and $a_{+}, a_{-}$are restricted by (3.13) with $\delta=+,-$. Whenever $a_{+} / a_{-}$is irrational, the restrictions (3.13) are obviously satisfied for all $N_{+}, N_{-} \in \mathbb{N}$. But (3.13) is also compatible with $a_{+} / a_{-} \in Q$ and a finite number of $\left(N_{+}, N_{-}\right) \in \mathbb{N}^{2}$. To be specific, letting 


$$
a_{+} / a_{-}=n_{-} / n_{+}, \quad n_{+}, n_{-} \in \mathbb{N}^{*}, \quad n_{+}, n_{-} \text {coprime, }
$$

one easily verifies that (3.13) with $\delta=+,-$ is satisfied if and only if

$$
N_{\delta} \in \mathbb{N}, \quad N_{\delta}<n_{\delta} / 2, \quad \delta=+,-
$$

Of course, for rational $a_{+} / a_{-}$there are infinitely many distinct pairs $\left(N_{+}, N_{-}\right) \in \mathbb{N}^{2}$ yielding the same $b$. But the conditions (3.13) cannot be satisfied for more than one pair. (Indeed, assuming, for instance, that

$$
(M+1) a_{+}-N a_{-}=\left(M^{\prime}+1\right) a_{+}-N^{\prime} a_{-}, \quad M>M^{\prime}>0,
$$

one gets $\left(M-M^{\prime}\right) a_{+}=\left(N-N^{\prime}\right) a_{-}$, so that $k a_{+} \in \mathbb{N} a_{-}$for some $k \in\{1, \ldots, M-1\}$.) This entails, in particular, that all of the numbers $\left(N_{+}+1\right) a_{+}-N_{-} a_{-}$arising from (3.27) and (3.28) are distinct.

Next, we consider the case

$$
b=\left(N_{-}+1\right) a_{-}-N_{+} a_{+}, \quad N_{+}, N_{-} \in \mathbb{N}^{*} .
$$

Clearly, this case can be handled in the same way as the case (3.1). Specifically, we need only interchange all subscripts + and - in various formulas, for example, in (3.2)-(3.4). Combining (3.4), (1.25), (1.27), and their obvious counterparts for (3.30), we obtain in both cases the same function $\mathcal{F}(x, y)(1.29)$, namely,

$$
\begin{aligned}
\mathcal{F}(x, y)= & \phi(\mathcal{N}) \prod_{\delta=+,-} \prod_{j=1}^{N_{\delta}} \frac{s_{-\delta}\left(x+z_{j}^{\delta}(y)\right)}{\left[s_{-\delta}\left(x+i j a_{\delta}\right) s_{-\delta}\left(x-i j a_{\delta}\right)\right]^{1 / 2}} \\
& \times \exp \left[\operatorname{irx}\left(2 N_{+} N_{-}+N_{+}+N_{-}+1\right)\right] \exp (i x y) .
\end{aligned}
$$

[Here, $\phi(\mathcal{N})$ is the phase of the normalization constant $\mathcal{N} ;$ cf. (1.24)-(1.29); these quantities can be explicitly calculated from Ref. 14 , but we do not need them in the elliptic case-by contrast to the hyperbolic case; cf. Sec. III in Ref. 11.] This coincidence is in agreement with the symmetry property (1.13) of the A $\Delta \mathrm{Os} H_{+}$and $H_{-}$(1.12). Note also that in the rational case the $b$-values obtained from (3.1) and (3.30) are distinct, save for one special case, viz., $n_{+}, n_{-}$odd; $N_{+}$ $=\left[n_{+} / 2\right], N_{-}=\left[n_{-} / 2\right]$, both in (3.1) and in (3.30).

In this section we have thus far excluded the special cases $N_{-}=0$ and/or $N_{+}=0$. But these cases can be easily handled, too. Indeed, when $N_{+}$and $N_{-}$both vanish, we may and will take

$$
\Psi(x, y)=\mathcal{N} \frac{1}{s_{-\delta}(x)} \exp (i r x+i x y), \quad b=a_{\delta}, \quad \delta=+,-,
$$

and when one of $N_{+}$and $N_{-}$equals 0 , we can proceed just as in Sec. II. Clearly, the resulting function $\mathcal{F}(x, y)(1.29)$ is given by (3.31) in these special cases, too.

We now summarize and extend the above findings in the following theorem.

Theorem III.1: Fix parameters in the set $\mathcal{D} \subset \mathcal{E}(1.14)$ defined by

$$
\begin{gathered}
b=\left(N_{\alpha}+1\right) a_{\alpha}-N_{-\alpha} a_{-\alpha}, \quad \alpha \in\{+,-\}, \quad N_{+}, N_{-} \in \mathbb{N}, \\
k a_{+} \notin \mathbb{N} a_{-}, \quad k=1, \ldots, 2 N_{+} \quad\left(N_{+}>0\right), \\
k a_{-} \notin \mathbb{N} a_{+}, \quad k=1, \ldots, 2 N_{-} \quad\left(N_{-}>0\right) .
\end{gathered}
$$

Then there exists $K \in \mathbb{R}$ such that for all $y \in(K, \infty)$ the following holds true.

(i) The above functions 


$$
z_{1}^{+}, \ldots, z_{N_{+}}^{+}, z_{1}^{-}, \ldots, z_{N_{-}}^{-}:(K, \infty) \rightarrow i(0, \infty)
$$

are real-analytic solutions to the system of equations (3.5), (3.10), and (3.11) (where $\delta=+,-$ and $\left.k=1, \ldots, N_{-}\right)$; they satisfy (3.19) and have large-y asymptotics

$$
z_{l}^{\delta}(y)=i l a_{\delta}+O\left(\exp \left(-2 l a_{\delta} y\right)\right), \quad l=1, \ldots, N_{\delta}, \quad \delta=+,-, \quad y \rightarrow \infty .
$$

(ii) The ADOs $H_{+}$and $H_{-}$(1.12) have joint eigenfunctions $\mathcal{F}( \pm x, y)$ given by (3.31), with eigenvalues $E_{+}(y)$ and $E_{-}(y)$ given by (3.20).

(iii) The eigenvalues are real-valued, real-analytic functions on $(K, \infty)$ satisfying (3.24), (3.26), and (1.20).

(iv) The $A \Delta O S A_{+}$and $A_{-}$(1.15) have joint eigenfunctions $\Psi( \pm x, y)$ with eigenvalues $E_{+}(y)$ and $E_{-}(y)$; explicitly,

$$
\Psi(x, y)=\mathcal{N} \prod_{j=-N_{\alpha}}^{N_{\alpha}} \frac{1}{s_{-\alpha}\left(x+i j a_{\alpha}\right)} \cdot \mathcal{H}(x, y),
$$

where

$$
\mathcal{H}(x, y) \equiv \prod_{\delta=+,-} \prod_{j=1}^{N_{\delta}} s_{-\delta}\left(x+z_{j}^{\delta}(y)\right) \cdot \exp \left[\operatorname{irx}\left(2 N_{+} N_{-}+N_{+}+N_{-}+1\right)+i x y\right]
$$

(v) Setting

$$
\mathcal{H}^{(\infty)}(x, y) \equiv \prod_{\delta=+,-} \prod_{j=1}^{N_{\delta}} s_{-\delta}\left(x+i j a_{\delta}\right) \cdot \exp \left[\operatorname{irx}\left(2 N_{+} N_{-}+N_{+}+N_{-}+1\right)+i x y\right],
$$

one has

$$
\mathcal{H}(x, y)=\mathcal{H}^{(\infty)}(x, y)+O\left(\exp \left(-2 \min \left(a_{+}, a_{-}\right) y\right)\right), \quad y \rightarrow \infty,
$$

where the bound is uniform on $x$-compacts.

(vi) The poles on the imaginary axis of the function

$$
\chi(x, y) \equiv \Psi(x, y)+\Psi(-x, y)
$$

are simple and can be located only at the points

$$
x=i k_{\alpha} a_{\alpha}+i k_{-\alpha} a_{-\alpha}, \quad \pm k_{\alpha} \in\left\{0,1, \ldots, N_{\alpha}\right\}, \quad \pm k_{-\alpha} \in\left\{N_{-\alpha}+1, N_{-\alpha}+2, \ldots\right\} .
$$

Proof: We have already proved (i)-(iv). The uniform large-y asymptotics (3.41) easily follows from (3.37). Thus, it remains to prove (vi). To this end we begin by generalizing the identities (2.35). Specifically, we claim that the function $\mathcal{H}(x, y)$ satisfies

$$
\mathcal{H}\left(i k_{+} a_{+}+i k_{-} a_{-}, y\right)=\mathcal{H}\left(-i k_{+} a_{+}-i k_{-} a_{-}, y\right), \quad \pm k_{\delta} \in\left\{0, \ldots, N_{\delta}\right\}, \quad \delta=+,-
$$

To prove this claim, we use (1.10) and (3.10) to write

$$
\mathcal{H}\left(i k_{+} a_{+}+i k_{-} a_{-}, y\right)=p\left(k_{+}, k_{-}\right) \prod_{\delta=+,-} \mathcal{H}_{N_{\delta}}^{\delta}\left(i k_{\delta} a_{\delta}\right), \quad k_{+}, k_{-} \in \mathbb{Z}
$$

Here, we have introduced 


$$
\mathcal{H}_{N_{\delta}}^{\delta}(x) \equiv \prod_{j=1}^{N_{\delta}} s_{-\delta}\left(x+z_{j}^{\delta}\right) \cdot \exp \left(\frac{x}{2 i a_{\delta}} \ln \left(\prod_{j=1}^{N_{\delta}} \frac{s_{-\delta}\left(z_{j}^{\delta}-i a_{\delta}\right)}{s_{-\delta}\left(z_{j}^{\delta}+i a_{\delta}\right)}\right)\right),
$$

and the prefactor reads

$$
p\left(k_{+}, k_{-}\right)=\prod_{\delta=+,-}(-)^{N_{\delta} k_{-}} \exp r N_{\delta}\left(k_{-\delta}^{2} a_{-\delta}+2 k_{+} k_{-} a_{\delta}\right)
$$

Now in (3.46) the $y$-dependence occurs via the $t_{\delta}$-dependence of $z_{j}^{\delta}$; cf. (3.18). We may therefore invoke our previous result (2.35) to deduce that our claim (3.44) holds true. (Note that $p$ is invariant under taking $k_{+}, k_{-} \rightarrow-k_{+},-k_{-}$.)

We now exploit the identities (3.44) to locate the poles of $\chi(x, y)$ on the imaginary axis. The product in (3.38) gives rise to poles at

$$
x=i k_{\alpha} a_{\alpha}+i k_{-\alpha} a_{-\alpha}, \quad \pm k_{\alpha} \in\left\{0, \ldots, N_{\alpha}\right\}, \quad k_{-\alpha} \in \mathbb{Z} .
$$

In view of (3.34) and (3.35), all of these poles are simple. Now for $\pm k_{-\alpha} \in\left\{0, \ldots, N_{-\alpha}\right\}$, the poles are matched by zeros of $\mathcal{H}(x, y)-\mathcal{H}(-x, y)$ due to (3.44). Therefore, poles of $\chi$ on the imaginary axis must be located at (3.43).

It is quite likely that $\chi(x, y)$ does have poles at the points (3.43), i.e., no further cancellation takes place. It is illuminating to rewrite these points as [cf. (3.33)]

$$
\begin{gathered}
\pm i x=b-a_{+}-a_{-}-k_{\alpha} a_{\alpha}-k_{-\alpha} a_{-\alpha}, \quad k_{\alpha} \in\left\{0, \ldots, N_{\alpha}\right\}, \quad k_{-\alpha} \in \mathbb{N}, \\
\pm i x=k_{\alpha} a_{\alpha}+\left(N_{-\alpha}+k_{-\alpha}\right) a_{-\alpha}, \quad k_{\alpha} \in\left\{0, \ldots, N_{\alpha}\right\}, \quad k_{-\alpha} \in \mathbb{N}^{*} .
\end{gathered}
$$

Indeed, from this representation it is clear that when $b$ takes values in a bounded subset of $\mathrm{R}$, then the number of poles in a rectangle $|\operatorname{Re} x|<\pi / r,|\operatorname{Im} x|<L$ is bounded above. Moreover, assuming $a_{+} / a_{-} \notin \mathrm{Q}$, the restrictions (3.34) and (3.35) hold for arbitrary $N_{+}, N_{-} \in \mathrm{N}^{*}$. Thus we may let $N_{+}, N_{-} \rightarrow \infty$, whilst keeping $b$ bounded. Doing so, the points (3.50) diverge away, whereas the points (3.49) become

$$
\pm i x=b-a_{+}-a_{-}-k_{+} a_{+}-k_{-} a_{-}, \quad k_{+}, k_{-} \in \mathbb{N} .
$$

The latter limits illuminate the issue of arbitrary- $b$ interpolations discussed already in Sec. I, but, of course, they do not imply that an interpolation exists. For one thing, the two summands of $\chi(x, y)$ have different Floquet multipliers $\exp ( \pm i \pi y / r)$ under $x \rightarrow x+\pi / r$ unless $y$ equals $n r, n$ $\in \mathbb{Z}$, so that generically no pole/zero cancellation occurs on the lines $\operatorname{Re} x=k \pi / r, k \in \mathbb{Z}^{*}$. Thus poles get dense on these lines as $N_{+}, N_{-} \rightarrow \infty$, and so a meromorphic interpolation is not likely to exist for generic $y$.

Specializing, however, to

$$
\chi_{n}(x) \equiv \chi(x, n r), \quad n r>K, \quad n \in \mathbb{N}
$$

we deduce

$$
\chi_{n}(x+\pi / r)=(-)^{n} \chi_{n}(x),
$$

so no such obstruction occurs for these functions. But we have neither information concerning parameter continuity nor any uniform bounds available that would help in proving the existence of a meromorphic interpolation.

Before studying orthogonality properties of the functions $\chi_{n}(x)$, we should consider the contingency that the functions $\Psi(x, y)$ and $\Psi(-x, y)$ are linearly dependent. Now they are manifestly not identically zero, and they have different Floquet multipliers unless $y$ equals $n r, n \in Z$, so we need only study whether one can have 


$$
\prod_{\delta=+,-} \prod_{j=1}^{N_{\delta}} \frac{s_{-\delta}\left(x+z_{j}^{\delta}(n r)\right)}{s_{-\delta}\left(x-z_{j}^{\delta}(n r)\right)}=C \exp \left[-2 i r x\left(2 N_{+} N_{-}+N_{+}+N_{-}+1+n\right)\right],
$$

where $C$ is a nonzero constant. Clearly, this equality can only hold if the poles and zeros on the lhs cancel. Recalling the asymptotics (3.34) and (3.35), one readily infers that no cancellation takes place for $n$ large enough.

For arbitrary $n r \in(K, \infty)$, however, we only know that we have $z_{j}^{\delta}(n r) \in i(0, \infty)$. (Recall that we have restricted $K$ such that this is the case.) Since the solution curve gets quite inaccessible when $y$ moves away from $\infty$, it appears hard to exclude pole/zero cancellation, in general. In view of the $\mathrm{A} \Delta \mathrm{E}$ (1.10), such a cancellation might be compatible with the rhs of (3.54).

Now, even without this difficulty, we know next to nothing about the minimal $K$ compatible with the various restrictions we have imposed. Thus, we may just as well assume that $K$ is chosen large enough so that for all $y \in(K, \infty)$ the functions $\Psi(x, y)$ and $\Psi(-x, y)$ are linearly independent, and we will do so from now on. Observe that this entails, in particular, that the functions $\chi_{n}(x)(3.52)$ do not vanish identically.

We conclude this section with some observations concerning parameter symmetries. To this end we fix $r, a_{+}, a_{-}>0$ and $N_{+}, N_{-} \in \mathbb{N}$ such that (3.34) and (3.35) hold true. Defining $b_{+}$ $\equiv\left(N_{+}+1\right) a_{+}-N_{-} a_{-}$, we then obtain a point $\left(r, a_{+}, a_{-}, b_{+}\right) \in \mathcal{D}$. Since the representation of $b_{+}$is unique [recall the paragraph containing (3.29)], we may define $\mathcal{F}\left(r, a_{+}, a_{-}, b_{+} ; x, y\right)$ by the rhs of (3.31). Likewise, setting $b_{-} \equiv\left(N_{-}+1\right) a_{-}-N_{+} a_{+}$yields a point in $\mathcal{D}$, and we may once more define $\mathcal{F}\left(r, a_{+}, a_{-}, b_{-} ; x, y\right)$ by the rhs of (3.31).

Proceeding in this way, we therefore obtain a well-defined function $\mathcal{F}\left(r, a_{+}, a_{-}, b ; x, y\right)$ for all points in $\mathcal{D}$. A moment's thought shows that this function obeys

$$
\begin{gathered}
\mathcal{F}\left(r, a_{+}, a_{-}, b ; x, y\right)=\mathcal{F}\left(r, a_{+}, a_{-}, a_{+}+a_{-}-b ; x, y\right), \\
\mathcal{F}\left(r, a_{+}, a_{-}, b ; x, y\right)=\mathcal{F}\left(r, a_{-}, a_{+}, b ; x, y\right) .
\end{gathered}
$$

Now the $w$-function $w\left(r, a_{+}, a_{-}, b ; x\right)$ is symmetric under $a_{+} \leftrightarrow a_{-}$, but not under $b \rightarrow a_{+}$ $+a_{-}-b$. Thus, setting

$$
\Psi\left(r, a_{+}, a_{-}, b ; x, y\right)=w\left(r, a_{+}, a_{-}, b ; x\right)^{-1 / 2} \mathcal{F}\left(r, a_{+}, a_{-}, b ; x, y\right)
$$

[cf. (1.29)], we are left with the symmetry

$$
\Psi\left(r, a_{+}, a_{-}, b ; x, y\right)=\Psi\left(r, a_{-}, a_{+}, b ; x, y\right) .
$$

Note that these symmetry properties for parameters in $\mathcal{D}$ have a bearing on eventual interpolations for parameters in $\mathcal{E}$.

\section{ORTHOGONALITY AND SELF-ADJOINTNESS ISSUES}

The functions $\chi_{n}$ (3.52) span an infinite-dimensional closed subspace

$$
\mathcal{H}_{w}(K) \subset \mathcal{H}_{w} \equiv L^{2}((0, \pi / r), w(x) d x) .
$$

The $\mathrm{A} \Delta \mathrm{Os} A_{\delta}$ give rise to densely defined operators in $\mathcal{H}_{w}(K)$ (denoted again by $A_{\delta}$ ) by setting

$$
A_{\delta} \chi_{n} \equiv E_{\delta}(n r) \chi_{n}, \quad n>K / r, \quad \delta=+,-,
$$

and extending linearly. The question of whether the operators thus obtained are symmetric amounts to the question of whether the functions $\chi_{n}$ are pairwise orthogonal. Indeed, orthogonality obviously entails symmetry, and symmetry entails orthogonality, since the eigenvalues are real and satisfy (1.20). 
We are now going to prove symmetry of the operators $A_{\delta}$ (and hence pairwise orthogonality of the functions $\chi_{n}$ ), assuming that the parameters belong to the convergence region $\mathcal{C}(1.42)$. This restriction is equivalent to

$$
\left(N_{\delta}+1\right) a_{\delta}>N_{-\delta} a_{-\delta}, \quad \delta=+,-,
$$

both when $\alpha=+$ and when $\alpha=-$ in (3.33).

Now when we work with the A $\Delta$ Os $A_{\delta}$ (1.15), then the cases $\alpha=+$ and $\alpha=-$ cannot be simultaneously handled, since $A_{+}$and $A_{-}$lack invariance under taking $b \rightarrow a_{+}+a_{-}-b$; cf. also (3.2) and (3.3). This asymmetry arises from the similarity transformation (1.16). Indeed, the $\mathrm{A} \Delta \mathrm{Os}$ $H_{\delta}(1.12)$ are invariant; cf. (1.13), but the $w$-function is not; cf. (1.45)-(1.47).

On the other hand, we may just as well work with the A $\Delta \mathrm{Os} H_{+}$and $H_{-}$and eigenfunctions $\Phi_{n}(x)$ (1.40), since (1.48) and (1.49) are unitarily equivalent to (4.1) and (4.2). But this choice has the drawback that square root branch points occur.

We shall therefore opt for a third unitarily equivalent setting that can be associated to parameters in $\mathcal{D}$. It gives rise to economic notation and meromorphic functions, and yields the same objects for the two choices of $\alpha$. Specifically, in view of (3.38) we may also consider the functions

$$
\psi_{n}(x) \equiv \mathcal{H}(x, n r)-\mathcal{H}(-x, n r)
$$

which yield a closed subspace

$$
\mathcal{H}_{\hat{w}}(K) \subset \mathcal{H}_{\hat{w}} \equiv L^{2}((0, \pi / r), \hat{w}(x) d x),
$$

with

$$
\begin{aligned}
\hat{w}(x) & \equiv(-)^{N_{+}+N_{-}+1} \mathcal{N}^{2} \prod_{j=-N_{\alpha}}^{N_{\alpha}} s_{-\alpha}\left(x+i j a_{\alpha}\right)^{-2} \cdot w\left(\left(N_{\alpha}+1\right) a_{\alpha}-N_{-\alpha} a_{-\alpha} ; x\right) \\
& =1 / \prod_{\delta=+,-} \prod_{ \pm=1}^{N_{\delta}} s_{-\delta}\left(x+i k a_{\delta}\right) ;
\end{aligned}
$$

cf. (1.41). The associated $\mathrm{A} \Delta \mathrm{O}$ s/operators on $\mathcal{H}_{\hat{w}}(K)$ are then given by

$$
B_{\delta}=p_{\delta}\left(\exp \left(-2 i r N_{\delta} x\right) \frac{s_{\delta}\left(x+i N_{-\delta} a_{-\delta}\right)}{s_{\delta}(x)} T_{i a_{-\delta}}+(i \rightarrow-i)\right), \quad \delta=+,- \text {, }
$$

where the prefactor reads

$$
p_{\delta} \equiv(-)^{N_{\delta}} \exp \left[a_{\delta} r\left(N_{\delta}+1\right) N_{\delta}-a_{-\delta} r\left(2 N_{\delta}+1\right)\left(N_{-\delta}+1\right)\right]
$$

[Recall that (3.2) and (3.3) hold for $\alpha=+$; cf. (3.1); the $\alpha=-$ counterparts are obtained by interchanging all subscripts + and - .]

Though the simplicity of this choice is already apparent, it should be emphasized that the weight function $\hat{w}(x)$ does not have a continuous extension to parameters in $\mathcal{E}(1.14)$, by contrast to $w(x)$. This can be seen, e.g., as follows. Fix $N_{+}, N_{-} \in \mathbb{N}^{*}$, and $a_{+}=a \in(0, \infty)$, and choose $b=\left(N_{+}+1\right) a-N_{-} a_{-}$. Now let $a_{-} \rightarrow q a$ with $q$ a positive rational number. Then $\hat{w}(x)$. (4.6) clearly has a well-defined limit. But there are infinitely many distinct pairs $k, l \in \mathbb{N}^{*}$ yielding the same $b$ for $a_{-} \rightarrow q a$ [i.e., such that $k a-l q a$ equals $\left(N_{+}+1\right) a-N_{-} q a$ ]. Evidently, each of these pairs yields a different limiting $\hat{w}(x)$.

Likewise, an interpolation obstruction is present for the $\mathrm{A} \Delta \mathrm{O}$ s $B_{\delta}$. [Choose, e.g., $N_{-}=N_{+}$ +1 and $q=1$ in the previous paragraph. From (4.7) one then sees that the limiting $b=0 \mathrm{~A} \Delta \mathrm{Os}$ 
depend on $N_{+}$; cf. (1.10).] Of course, this leads one to expect that the joint eigenfunctions $\mathcal{H}( \pm x, y)$ cannot be interpolated either. In the hyperbolic context we show that this expectation is indeed fulfilled; cf. Sec. III in Ref. 11.

As long as we restrict attention to a fixed choice of parameters in $\mathcal{D}$ though, the third setting just detailed is the simplest to study. We shall also use it in Appendix B, where we prove that any meromorphic joint eigenfunction $M(x)$ with $B_{\delta}$-eigenvalues $E_{\delta}(y), \delta=+,-$, must be a linear combination of $\mathcal{H}(x, y)$ and $\mathcal{H}(-x, y)$, provided $a_{+} / a_{-}$is irrational and $y \in(L, \infty)$ for some $L$ $\geqslant K$.

Returning now to the symmetry question, we begin by observing that the functions $\psi_{n}(x)$ (4.4) are entire, odd, and $2 \pi / r$-periodic. Moreover, provided $|k| \leqslant N_{+},|l| \leqslant N_{-}$, they have zeros in the points

$$
z_{k l} \equiv i k a_{+}+i l a_{-}, \quad k, l \in \mathbb{Z},
$$

[due to (3.44)], and in the points $z_{k l}+\pi / r$ (since they are either $\pi / r$-periodic or $\pi / r$-antiperiodic).

Let us now define the vector spaces

$$
\begin{gathered}
\mathcal{O}_{0} \equiv\{F(x) \text { entire, odd, } 2 \pi / r \text {-periodic }\}, \\
\mathcal{O}_{1} \equiv\left\{F \in \mathcal{O}_{0}\left|F\left(z_{k l}\right)=0, \quad F\left(z_{k l}+\pi / r\right)=0, \quad\right| k\left|\leqslant N_{+},\right| l \mid \leqslant N_{-}\right\}, \\
\mathcal{O}_{2} \equiv\left\{F \in \mathcal{O}_{0}\left|F\left(z_{k l}\right)=0, \quad F\left(z_{k l}+\pi / r\right)=0, \quad k \in \mathbb{Z},\right| l \mid \leqslant N_{-}, \quad \text { and }|k| \leqslant N_{+}, l \in \mathbb{Z}\right\} .
\end{gathered}
$$

Clearly, we have

$$
\mathcal{O}_{2} \subset \mathcal{O}_{1} \subset \mathcal{O}_{0} \subset \mathcal{H}_{\hat{w}}
$$

and

$$
\psi_{n} \in \mathcal{O}_{1}, \quad n>K / r .
$$

Next, we fix $F \in \mathcal{O}_{0}$ and consider $B_{+} F$. For $N_{-}=0$ we have $B_{+} F \in \mathcal{O}_{0}$, but for $N_{-}>0$ we get

$$
\lim _{x \rightarrow 0} s_{+}(x)\left(B_{+} F\right)(x)=2 p_{+} s_{+}\left(i N_{-} a_{-}\right) F\left(-i a_{-}\right),
$$

where we used (4.7) and oddness of $F$. Now the rhs does not vanish unless $F\left(\right.$ ia $\left.a_{-}\right)=0$, so, in general, $\left(B_{+} F\right)(x)$ has a pole at $x=0$, entailing $B_{+} F \notin \mathcal{H}_{\hat{w}}$. Assuming $F \in \mathcal{O}_{1}$, however, we have $F\left(i a_{-}\right)=0$ and $F\left(\pi / r+i a_{-}\right)=0$, so that $B_{+} F \in \mathcal{H}_{\hat{w}}$. More generally, this argument yields the conclusion

$$
B_{\delta} \mathcal{O}_{1} \subset \mathcal{H}_{\hat{w}}, \quad \delta=+,-
$$

Therefore, the $\mathrm{A} \Delta \mathrm{Os} B_{\delta}$ give rise to Hilbert space operators

$$
B_{\delta}^{(j)}: \mathcal{O}_{j} \rightarrow \mathcal{H}_{\hat{w}}, \quad F(x) \mapsto\left(B_{\delta} F\right)(x),
$$

where $\delta=+,-$ and $j=1,2$.

It is not hard to see that these operators are densely defined. Indeed, $\mathrm{O}_{2}$ contains the subspace

$$
\mathcal{O}_{\delta} \equiv \frac{s_{\delta}(x) s_{-\delta}(x)^{2}}{\hat{w}(x)} \operatorname{Pol}(\cos r x), \quad \delta \in\{+,-\}
$$

where $\operatorname{Pol}(t)$ denotes the space of polynomials in $t$, and $\mathcal{O}_{\delta}$ is clearly dense in $\mathcal{H}_{\hat{w}}$. The following theorem makes clear why it is important to distinguish between the operators $B_{\delta}^{(1)}$ and $B_{\delta}^{(2)}$. 
Theorem IV.1: The operators $B_{\delta}^{(2)}$ are symmetric for all parameters in $\mathcal{D}$; moreover, their adjoints extend the operators $B_{\delta}^{(1)}$. The operators $B_{\delta}^{(1)}$ are not symmetric for parameters in $\mathcal{D} \backslash \mathcal{C}$, whereas they are symmetric for parameters in $\mathcal{D} \cap \mathcal{C}$.

Proof: For convenience we choose $\delta=-;$ the case $\delta=+$ can then be handled by interchanging the subscripts + and - in the following. To prove symmetry of $B_{-}^{(2)}$, it suffices to show $I_{L}$ $=I_{R}$, with

$$
\begin{gathered}
I_{L} \equiv \int_{0}^{\pi / r}\left(\exp \left(2 i r N_{-} x\right) \frac{s_{-}\left(x-i N_{+} a_{+}\right)}{s_{-}(x)} F^{*}\left(x+i a_{+}\right)+(i \rightarrow-i)\right) G(x) \hat{w}(x) d x, \\
I_{R} \equiv \int_{0}^{\pi / r} F^{*}(x)\left(\exp \left(-2 i r N_{-} x\right) \frac{s_{-}\left(x+i N_{+} a_{+}\right)}{s_{-}(x)} G\left(x-i a_{+}\right)+(i \rightarrow-i)\right) \hat{w}(x) d x,
\end{gathered}
$$

where we take $F, G \in \mathcal{O}_{2}$, and where we employ the notation

$$
F^{*}(x) \equiv \overline{F(\bar{x})}
$$

In order to prove equality of these integrals, we introduce the function

$$
I(x) \equiv \hat{w}(x-e) \exp \left(2 i r N_{-}(x-e)\right) \frac{s_{-}\left(x-e-i N_{+} a_{+}\right)}{s_{-}(x-e)} F^{*}(x+e) G(x-e), \quad e \equiv \frac{i a_{+}}{2} .
$$

From the definition (4.6) of $\hat{w}(x)$ we deduce that we may rewrite $I(x)$ as

$$
I(x)=\hat{w}(x+e) \exp \left(-2 i r N_{-}(x+e)\right) \frac{s_{-}\left(x+e+i N_{+} a_{+}\right)}{s_{-}(x+e)} F^{*}(x+e) G(x-e) .
$$

Now $\hat{w}(x)$ is even and $s_{-}(x), F^{*}(x)$ and $G(x)$ are odd, so we have

$$
\begin{aligned}
I_{L}-I_{R} & =\int_{0}^{\pi / r}(I(x+e)+I(-x+e)-I(x-e)-I(-x-e)) d x \\
& =\int_{-\pi / r}^{\pi / r}(I(x+e)-I(x-e)) d x, \quad e \equiv i a_{+} / 2 .
\end{aligned}
$$

Recalling $F^{*}$ and $G$ are $2 \pi / r$-periodic and noting $\hat{w}$ is $\pi / r$-periodic, it follows that $I(x)$ is $2 \pi / r$-periodic. Thus the integral (4.24) vanishes (by Cauchy's theorem) whenever $I(x)$ has no poles in the strip $|\operatorname{Im} x| \leqslant a_{+} / 2$. Now since we assumed $F, G \in \mathcal{O}_{2}$, the function $I(x)$ is, in fact, entire. Thus we obtain $I_{L}=I_{R}$, and so $B_{-}^{(2)}$ is indeed symmetric.

Choosing next $F, G \in \mathcal{O}_{1}$, we can proceed in the same way as before, but now $I(x)$ has poles, in general. But when one of $F, G$ belongs to $\mathcal{O}_{2}$, then one easily sees that $I(x)$ is still entire. Thus, the domain of $B_{-}^{(2) *}$ contains $\mathcal{O}_{1}$, and the action of $B_{-}^{(2) *}$ on $\mathcal{O}_{1}$ coincides with the action of the $\mathrm{A} \Delta \mathrm{O} B_{-}$. A moment's thought now shows that this state of affairs holds true on a larger subspace than $\mathcal{O}_{1}$, so that the adjoint of $B_{-}^{(2)}$ is a proper extension of $B_{-}^{(1)}$. (One need not require entireness, for instance.)

To prove the second assertion of the theorem, we determine the location of eventual poles of $I(x)$ for $F, G \in \mathcal{O}_{1}$. From (4.22) and (4.6) we infer that the poles of $I(x)$ are equal to the poles of the function

$$
J(x) \equiv\left(\prod_{ \pm k=1}^{N_{-}} s_{+}\left(x-e+i k a_{-}\right)\right)^{-1}\left(\prod_{n=-N_{+}+1 / 2}^{N_{+}-1 / 2} s_{-}\left(x+i n a_{+}\right)\right)^{-1} F^{*}(x+e) G(x-e) .
$$


Let us first analyze the poles of $J(x)$ on the imaginary axis, using the notation

$$
p_{k n} \equiv i k a_{-}+i n a_{+}, \quad k \in \mathbb{Z}, \quad n \in \mathbb{Z}+1 / 2 .
$$

The first product yields poles at the points

$$
p_{k n}, \quad \pm k \in\left\{1, \ldots, N_{-}\right\}, \quad n \in \mathbb{Z}+1 / 2,
$$

and the second one at

$$
p_{k n}, \quad k \in \mathbb{Z}, \quad \pm n \in\left\{1 / 2, \ldots, N_{+}-1 / 2\right\} .
$$

Thus, the products yield double poles at

$$
p_{k n}, \quad \pm k \in\left\{1, \ldots, N_{-}\right\}, \quad \pm n \in\left\{1 / 2, \ldots, N_{+}-1 / 2\right\},
$$

and simple poles at

$$
\begin{gathered}
p_{k n}, \quad \pm k \in\left\{0, N_{-}+1, N_{-}+2, \ldots\right\}, \quad \pm n \in\left\{1 / 2, \ldots, N_{+}-1 / 2\right\}, \\
p_{k n}, \quad \pm k \in\left\{1, \ldots, N_{-}\right\}, \quad \pm n \in\left\{N_{+}+1 / 2, N_{+}+3 / 2, \ldots\right\} .
\end{gathered}
$$

Now the function $F^{*}(x+e) G(x-e)$ has double zeros at

$$
p_{k n}, \quad \pm k \in\left\{0, \ldots, N_{-}\right\}, \quad \pm n \in\left\{1 / 2, \ldots, N_{+}-1 / 2\right\},
$$

and simple zeros at

$$
p_{k n}, \quad \pm k \in\left\{0, \ldots, N_{-}\right\}, \quad \pm n=N_{+}+1 / 2 .
$$

Therefore, poles of $J(x)$ can be located solely at the points

$$
\begin{gathered}
p_{k n}, \quad \pm k \in\left\{N_{-}+1, N_{-}+2, \ldots\right\}, \quad \pm n \in\left\{1 / 2, \ldots, N_{+}-1 / 2\right\}, \\
p_{k n}, \quad \pm k \in\left\{1, \ldots, N_{-}\right\}, \quad \pm n \in\left\{N_{+}+3 / 2, N_{+}+5 / 2, \ldots\right\} .
\end{gathered}
$$

We proceed by proving that for parameters in $\mathcal{D} \cap \mathcal{C}$ the latter points lie outside the strip $|\operatorname{Im} x| \leqslant a_{+} / 2$. Consider first (4.34). When $k$ and $n$ have the same sign, it is immediate that these points are outside the critical strip. Now let $k>0$ and $n<0$. Then we get

$$
k a_{-}+n a_{+} \geqslant\left(N_{-}+1\right) a_{-}-\left(N_{+}-1 / 2\right) a_{+}>a_{+} / 2,
$$

due to (4.3). Similarly, we have $k a_{-}+n a_{+}<-a_{+} / 2$ for $k<0$ and $n>0$. Next, consider (4.35). Taking $k>0$ and $n<0$, we now have

$$
k a_{-}+n a_{+} \leqslant N_{-} a_{-}-\left(N_{+}+3 / 2\right) a_{+}<-a_{+} / 2,
$$

due to (4.3); the other cases are then clear.

The upshot is that eventual poles of $J(x)$ on the imaginary axis lie outside $|\operatorname{Im} x| \leqslant a_{+} / 2$. The above analysis can be repeated for poles on the line $\operatorname{Re} x=\pi / r$, yielding the same conclusion. Since $J(x)$ is $2 \pi / r$-periodic, we deduce the absence of poles in the critical strip. Thus $I(x)$ has no poles in the strip either, and so the integral (4.24) vanishes. Hence, $B_{-}^{(1)}$ is symmetric when (4.3) holds true.

Finally, we choose parameters in $\mathcal{D} \backslash \mathcal{C}$, so that (4.3) is violated. Thus, we either have $\left(N_{-}\right.$ $+1) a_{-}<N_{+} a_{+}$or $\left(N_{+}+1\right) a_{+}<N_{-} a_{-}$. In the first case we have

$$
\left(N_{-}+1\right) a_{-}-\left(N_{+}-1 / 2\right) a_{+}<a_{+} / 2, \quad\left(N_{-}+1\right) a_{-}-a_{+} / 2>-a_{+} / 2,
$$


so at least one of the points $p_{N_{-}+1, n}(4.34)$ is in the critical strip. In the second case we have

$$
N_{-} a_{-}-\left(N_{+}+3 / 2\right) a_{+}>-a_{+} / 2
$$

so at least one of the points $p_{N_{-}, n}$ (4.35) is in the strip. In either case, the integral (4.24) does not vanish, in general, since we are free to choose the values of $F$ and $G$ in the pertinent points. Therefore, $B_{-}^{(1)}$ is not symmetric for parameters outside the convergence region $\mathcal{C}$.

Taking $F=\psi_{n}, G=\psi_{m}$ in the proof of this theorem, we clearly have

$$
I_{L}-I_{R}=\left[E_{-}(n r)-E_{-}(m r)\right]\left(\psi_{n}, \psi_{m}\right) .
$$

For parameters in $\mathcal{C}$ we therefore conclude that [using (1.20)]

$$
\left(\psi_{n}, \psi_{m}\right)=0, \quad n>m>K / r .
$$

But for parameters outside $\mathcal{C}$ we cannot prove that (4.41) is violated. The point is that the relevant residue $\operatorname{sum}(\mathrm{s})$ might vanish.

We conjecture that this does not happen in general. More precisely, fixing parameters in $\mathcal{D} \backslash \mathcal{C}$, we expect that one can find a pair $n \neq m$ such that $\left(\psi_{n}, \psi_{m}\right) \neq 0$. Choosing $b=2 a_{\alpha}$, this conjectured orthogonality breakdown can be explicitly verified for $a_{\alpha}>a_{-\alpha}$ (with $a_{\alpha} \notin \mathbb{N} a_{-\alpha} / 2$ ) and all pairs $n \neq m$ with $n-m$ even. Indeed, in this special case the integral (4.24) with $F=\psi_{n}, G=\psi_{m}$ equals a nonzero residue sum. [The resulting formula for $\left(\psi_{n}, \psi_{m}\right)$ amounts to the formula obtained by more direct means in Ref. 12 , so we skip the details.]

Since the $\mathrm{A} \Delta \mathrm{Os} B_{\delta}$ and domains $\mathcal{O}_{j}$ are invariant under complex conjugation, the operators $B_{\delta}^{(j)}$ admit self-adjoint extensions whenever they are symmetric. Fixing parameters in $\mathcal{C}$, the operators $B_{\delta}^{(1)}$ are most likely essentially self-adjoint, but the state of affairs for $B_{\delta}^{(2)}$ is quite opaque to us. We add one observation on the self-adjoint extensions of the latter, however. Whenever one chooses parameters outside $\mathcal{C}$ and a pair $n \neq m$ with $\left(\psi_{n}, \psi_{m}\right) \neq 0$, any self-adjoint extension of $B_{\delta}^{(2)}$ has a domain to which $\psi_{n}$ and $\psi_{m}$ may or may not belong, but if both functions belong to the domain, then the action of the extension on at least one of them cannot coincide with the $B_{\delta^{-}}$action. [If it did coincide, one would deduce $\left(\psi_{n}, \psi_{m}\right)=0$, a contradiction.]

Let us now return to the subspace $\mathcal{H}_{w}(K)(4.1)$ and operators $A_{\delta}$ (4.2). For parameters outside $\mathcal{C}$, the operators $A_{\delta}$ are not symmetric whenever a pair $n \neq m$ exists for which $\left(\chi_{n}, \chi_{m}\right) \neq 0$. As mentioned above, we believe that this is always the case. Choosing parameters in $\mathcal{C}$, however, (4.41) amounts to pairwise orthogonality of the functions $\chi_{n}, n>K / r$, so that the operators $A_{\delta}$ are symmetric, as announced. We also expect that for parameters in $\mathcal{C}$ the orthocomplement of $\mathcal{H}_{w}(K)$ is spanned by joint eigenfunctions $\chi_{0}, \ldots, \chi_{M}, M=[K / r]$, of the $\mathrm{A} \Delta \mathrm{Os} A_{\delta}$ with real eigenvalues. (If so, the $\mathrm{A} \Delta \mathrm{Os} A_{\delta}$ are essentially self-adjoint on the linear span of $\chi_{0}, \chi_{1}, \ldots$, of course.)

We conclude this section by exploiting the $A \Delta O s B_{\delta}$ (4.7) and their eigenfunctions $\mathcal{H}( \pm x, y)$ (3.39) in yet another way. Specifically, we use them to obtain and study joint eigenfunctions of the $\mathrm{A} \Delta \mathrm{Os} A_{\delta}(b)$ (1.15) for $b=-N_{+} a_{+}-N_{-} a_{-}$and for $b=\left(N_{+}+1\right) a_{+}+\left(N_{-}\right.$ $+1) a_{-}$. Here we have $N_{+}, N_{-} \in \mathbb{N}$, and $a_{+}, a_{-}$are restricted by (3.34) and (3.35).

Let us recall first that both for $b=\left(N_{+}+1\right) a_{+}-N_{-} a_{-}$and for $b=\left(N_{-}+1\right) a_{-}-N_{+} a_{+}$we obtain the same $\mathrm{A} \Delta \mathrm{Os} B_{\delta}$ and eigenfunctions $\mathcal{H}( \pm x, y)$. Thus, in both cases we may denote the $\mathrm{A} \Delta \mathrm{Os}$ by $B_{\delta}\left(N_{+}, N_{-}\right)$, and their eigenvalues and eigenfunctions by $E_{\delta}\left(N_{+}, N_{-} ; y\right)$ and $\mathcal{H}_{N_{+}, N_{-}}( \pm x, y)$. The key observation is now that we have the identities

$$
\begin{gathered}
A_{\delta}\left(-N_{+} a_{+}-N_{-} a_{-}\right)=r_{\delta} B_{\delta}\left(N_{+}, N_{-}\right), \quad \delta=+,-, \\
A_{\delta}\left(\left(N_{+}+1\right) a_{+}+\left(N_{-}+1\right) a_{-}\right)=P_{N_{+}, N_{-}}(x)^{-1} r_{\delta} B_{\delta}\left(N_{+}, N_{-}\right) P_{N_{+}, N_{-}}(x), \quad \delta=+,-,
\end{gathered}
$$




$$
\begin{gathered}
r_{\delta} \equiv \exp a_{-\delta} r\left(2 N_{+}+1\right)\left(2 N_{-}+1\right), \quad \delta=+,-, \\
P_{N_{+}, N_{-}}(x) \equiv \prod_{\delta=+,-} \prod_{j=-N_{\delta}}^{N_{\delta}} s_{-\delta}\left(x+i j a_{\delta}\right), \quad N_{+}, N_{-} \in \mathbb{N} .
\end{gathered}
$$

[Indeed, this can be verified directly from (1.15) and (4.7) by using the $\mathrm{A} \Delta \mathrm{E}$ (1.10).]

As a result, we deduce

$$
\begin{gathered}
A_{\delta}\left(-N_{+} a_{+}-N_{-} a_{-}\right) \mathcal{H}_{N_{+}, N_{-}}( \pm x, y)=r_{\delta} E_{\delta}\left(N_{+}, N_{-} ; y\right) \mathcal{H}_{N_{+}, N_{-}}( \pm x, y), \\
A_{\delta}\left(\left(N_{+}+1\right) a_{+}+\left(N_{-}+1\right) a_{-}\right) P_{N_{+}, N_{-}}(x)^{-1} \mathcal{H}_{N_{+}, N_{-}}( \pm x, y) \\
=r_{\delta} E_{\delta}\left(N_{+}, N_{-} ; y\right) P_{N_{+}, N_{-}}(x)^{-1} \mathcal{H}_{N_{+}, N_{-}}( \pm x, y) .
\end{gathered}
$$

Thus, we obtain the joint eigenfunctions announced above. We claim that the $y \rightarrow \infty$ asymptotics of these new eigenfunctions and eigenvalues ties in with the asymptotics for the dense parameter set $\mathcal{D}$. (Notice that the new parameters do not belong to $\mathcal{D}$.) More precisely, we claim that this holds true when we set

$$
\begin{gathered}
\Psi(x, y) \equiv \mathcal{N} H_{N_{+}, N_{-}}\left(x, y\left(N_{+}, N_{-}\right)\right), \quad b=-N_{+} a_{+}-N_{-} a_{-}, \\
\Psi(x, y) \equiv \mathcal{N} P_{N_{+}, N_{-}}(x)^{-1} \mathcal{H}_{N_{+}, N_{-}}\left(x, y\left(N_{+}, N_{-}\right)\right), \quad b=\left(N_{+}+1\right) a_{+}+\left(N_{-}+1\right) a_{-},
\end{gathered}
$$

where

$$
y\left(N_{+}, N_{-}\right) \equiv y-\left(2 N_{+}+1\right)\left(2 N_{-}+1\right) r
$$

so that the associated eigenvalues read

$$
E_{\delta}(y)=r_{\delta} E_{\delta}\left(N_{+}, N_{-} ; y\left(N_{+}, N_{-}\right)\right), \quad \delta=+,-.
$$

To prove this claim, we recall the $\mathcal{H}$-asymptotics given by (3.40) and (3.41). It entails that $\Psi(x, y)$ as just defined satisfies (1.24), where the $c$-function reads

$$
c\left(-N_{+} a_{+}-N_{-} a_{-} ; x\right)=\mathcal{N} \prod_{\delta=+,-} \prod_{j=1}^{N_{\delta}} s_{-\delta}\left(x+i j a_{\delta}\right) \cdot \exp -i r x\left(2 N_{+} N_{-}+N_{+}+N_{-}\right),
$$

$$
c\left(\left(N_{+}+1\right) a_{+}+\left(N_{-}+1\right) a_{-} ; x\right)=\mathcal{N} \prod_{\delta=+,-} \prod_{j=0}^{N_{\delta}} \frac{1}{s_{-\delta}\left(x-i j a_{\delta}\right)} \cdot \exp -i r x\left(2 N_{+} N_{-}+N_{+}+N_{-}\right) .
$$

The point is now that this agrees with the interpolation (1.26) for a suitable choice of $\mathcal{N}$ $=\mathcal{N}\left(r, a_{+}, a_{-}, b\right)$. (This readily follows from Proposition III.8 in Ref. 14.) Similarly, the eigenvalues (4.51) have once more the $y$-asymptotics (1.19), as is clear from the asymptotics of $E_{\delta}\left(N_{+}, N_{-} ; y\right)$ and the definition (4.44) of $r_{\delta}$.

It is easily checked that the new eigenfunctions $\Psi(x, y)(4.48)$ and (4.49) also satisfy the quasiperiodicity relations (1.36). Thus, they are eigenfunctions of the $\mathrm{A} \Delta \mathrm{O} Q$ (1.38) with eigenvalue $2 \cos (\pi y / r)$; cf. (1.39). Furthermore, choosing $a_{+} / a_{-}$irrational, the uniqueness result in Appendix $\mathrm{B}$ applies. All of these findings are consistent with the existence of interpolating meromorphic joint $\left(A_{+}, A_{-}, Q\right)$-eigenfunctions $\Psi(x, y)$ for parameters in $\mathcal{E}(1.14)$, but they show once more that such an interpolation must have striking analyticity properties.

For instance, taking $N_{+}=N_{-}=0$ in (4.48), we obtain 


$$
\Psi(x, y)=\exp i x y, \quad b=0 ;
$$

cf. (3.39), (1.24), and (1.26) with $b=0$. Now when we fix $a_{+}$and $a_{-}$with $a_{+} / a_{-}$irrational, and let the number $b \equiv\left(N_{+}+1\right) a_{+}-N_{-} a_{-}$converge to 0 (by taking $N_{+}, N_{-} \rightarrow \infty$ in a suitable way), then the poles of the associated functions $\Psi(x, y)$ (1.22) become dense on the lines $\operatorname{Im} x$ $=k \pi / r, k \in \mathbb{Z}$. It is fully unclear to us whether and how (suitable $y$-dependent multiples of) these functions converge to the entire function (4.54) as $b \rightarrow 0$. But if a continuous interpolation can be found, then the existence of this limit would be a corollary.

It should be noted that the $b$-values in (4.48) and (4.49) are outside the orthogonality region $\mathcal{C}$ (1.42). Of course, "orthogonality" refers to the Hilbert space $L^{2}((0, \pi / r), w(x) d x)$, with $w(x)=w\left(r, a_{+}, a_{-}, b ; x\right)$. Now from (1.27), (4.52), and (4.53) we have

$$
\begin{gathered}
w\left(-N_{+} a_{+}-N_{-} a_{-} ; x\right)=(-)^{N_{+}+N_{-}} \mathcal{N}^{-2} \hat{w}_{N_{+}, N_{-}}(x), \\
w\left(\left(N_{+}+1\right) a_{+}+\left(N_{-}+1\right) a_{-} ; x\right)=(-)^{N_{+}+N_{-}} \mathcal{N}^{-2} P_{N_{+}, N_{-}}(x)^{2} \hat{w}_{N_{+}, N_{-}}(x),
\end{gathered}
$$

where $\hat{w}_{N_{+}, N_{-}}(x)$ is given by (4.6). Therefore, the analysis embodied in Theorem IV.1 can be applied to the odd linear combinations,

$$
\zeta_{n}(x) \equiv \Psi(x, n r)-\Psi(-x, n r), \quad n \in \mathbb{N}, \quad n r>K+\left(2 N_{+}+1\right)\left(2 N_{-}+1\right) r,
$$

to deduce orthogonality whenever (4.3) is satisfied.

For the even combinations $\chi_{n}(x)$, though, this analysis renders it quite unlikely that orthogonality holds true. In fact, for the trigonometric specialization with $b=-N_{+} a_{+}, N_{+}>0$, we prove in Sec. IV of Ref. 11 that orthogonality is indeed violated. Thus, in the elliptic case this must also be generically true. [Of course, the two cases where $N_{+}=N_{-}=0$, namely $b=0$ and $b=a_{+}$ $+a_{-}$, are exceptional in this regard; cf. (4.54); observe that they correspond to the boundary of c.]

Finally, we point out that the functions $\widetilde{\mathcal{F}}(x, y)$ (1.29) for the new $b$-values $\left(N_{+}+1\right) a_{+}$ $+\left(N_{-}+1\right) a_{-}$and $-N_{+} a_{+}-N_{-} a_{-}$are in essence equal to the functions $\mathcal{F}(x, y)$ (3.31) for the $b$-values $\left(N_{+}+1\right) a_{+}-N_{-} a_{-}$and $\left(N_{-}+1\right) a_{-}-N_{+} a_{+}$. More precisely, we have

$$
\widetilde{\mathcal{F}}(x, y)=\chi \mathcal{F}\left(x, y\left(N_{+}, N_{-}\right)\right),
$$

where $\chi$ is a normalizing phase. [Indeed, this is readily verified by combining (4.48), (4.49) with (4.55), (4.56).]

This intimate relation [and also the formulas (4.42)-(4.51), for that matter] can be understood from a consideration of the $\mathrm{A} \Delta \mathrm{Os} H_{\delta}(b)$ given by (1.12). Indeed, it is straightforward to verify that one has the identity

$$
H_{\delta}\left(-N_{+} a_{+}-N_{-} a_{-}\right)=r_{\delta} H_{\delta}\left(\left(N_{+}+1\right) a_{+}-N_{-} a_{-}\right), \quad \delta=+,-,
$$

so the symmetry (1.13) also entails the identity

$$
H_{\delta}\left(\left(N_{+}+1\right) a_{+}+\left(N_{-}+1\right) a_{-}\right)=r_{\delta} H_{\delta}\left(\left(N_{+}+1\right) a_{+}-N_{-} a_{-}\right), \quad \delta=+,-.
$$

This explains why (4.58) holds: the relevant $\mathrm{A} \Delta \mathrm{O}$ s are proportional.

More generally, a consideration of the zeros of the coefficients of $H_{\delta}(b)$ shows that proportionality of $H_{\delta}\left(b_{1}\right)$ and $H_{\delta}\left(b_{2}\right)$ [for arbitrary $\left.a_{+}, a_{-} \in(0, \infty)\right]$ not only holds for $b_{2}=b_{1}$ (trivially) and $b_{2}=a_{+}+a_{-}-b_{1}$ [cf. (1.13)], but also when $b_{1}$ is of the quite special form

$$
2 b_{1}=k a_{+}+l a_{-}, \quad k, l \in \mathbb{Z},
$$

and $b_{2}$ satisfies 


$$
2 b_{2} \in\left\{k a_{+}+l a_{-}, k a_{+}+(-l+2) a_{-},(-k+2) a_{+}+l a_{-},(-k+2) a_{+}+(-l+2) a_{-}\right\} .
$$

Thus we are dealing with the case $k, l \in 2 \mathbb{Z}$ for the $b$-values at issue.

\section{ACKNOWLEDGMENT}

This work was partly supported by the Netherlands Organisation for the Advancement of Research (NWO).

\section{APPENDIX A: THE CONSTRAINT SYSTEM}

Let $f(w)$ be an entire, odd function satisfying

$$
f(w)=w+O\left(w^{3}\right), \quad w \rightarrow 0 .
$$

Fixing $N \geqslant 2$, define functions $F_{1}, \ldots, F_{N}$ by

$$
F_{k}: \mathrm{C}^{N} \rightarrow \mathrm{C}, \quad W \mapsto f\left(w_{k}-N\right) \prod_{\substack{j=1 \\ j \neq k}}^{N} f\left(w_{k}-w_{j}+1\right) \prod_{j=1}^{N} f\left(w_{j}+1\right)-(W \rightarrow-W) .
$$

Then we have the following result concerning the system of $N$ equations:

$$
F_{k}(W)=0, \quad k=1, \ldots, N,
$$

for $N$ unknowns $w_{1}, \ldots, w_{N}$.

Theorem A.1: The system (A3) admits the solution

$$
W_{0} \equiv(1,2, \ldots, N)
$$

and the determinant of the functional matrix

$$
(D F)_{k l} \equiv \partial_{l} F_{k}, \quad k, l=1, \ldots, N,
$$

vanishes for $W=W_{0}$. Assuming

$$
f(k) \neq 0, \quad k=1,2, \ldots, N+1,
$$

the system of $N-1$ equations

$$
F_{k}(W)=0, \quad k=2, \ldots, N,
$$

for $N$ unknowns $w_{1}, \ldots, w_{N}$ admits a unique solution of the form

$$
W(t)=\left(1+t, w_{2}(t), \ldots, w_{N}(t)\right),
$$

near $W_{0}$, with $w_{k}(t)$ holomorphic at $t=0$ and such that

$$
w_{k}(t)=k+O\left(t^{2}\right), \quad w_{k}^{\prime}(t)=O(t), \quad t \rightarrow 0, \quad k=2, \ldots, N .
$$

Moreover, assuming that $f(w)$ is real-valued for real $w$, the functions $w_{k}(t)$ are real-valued for $t \in(-\epsilon, \epsilon)$ and $\epsilon$ small enough.

Proof: Letting $W=W_{0}$, the second term on the rhs of (A2) vanishes, since $W_{0,1}=1$. The first term vanishes for $k=N$, since $W_{0, N}=N$. For $k<N$ the first term vanishes too, since $f\left(w_{k}-w_{j}\right.$ +1 ) yields a zero when $j=k+1$. Thus, $W_{0}$ solves the system (A3).

Next, we calculate the functional matrix $(D F)\left(W_{0}\right)$. Due to the factor $f\left(-w_{1}+1\right)$ in the second term on the rhs of (A2), this term can only yield a nonvanishing contribution to $\partial_{l} F_{k}\left(W_{0}\right)$ 
for $l=1$, and then the partial $\partial_{1}$ must act on $f\left(-w_{1}+1\right)$. Since $f\left(-w_{k}+w_{j}+1\right)$ yields a zero for $k=2, \ldots, N$ and $j=k-1$, the second term only contributes to $\left(\partial_{1} F_{1}\right)\left(W_{0}\right)$. Specifically, using $f^{\prime}(0)=1[$ cf. (A1)] we get

$$
\begin{aligned}
\left(\partial_{1} F_{1}\right)\left(W_{0}\right) & =f(1-N) \prod_{j=3}^{N} f(-j+2) \prod_{j=1}^{N} f(j+1)+f(-1-N) \prod_{j=2}^{N} f(j) \prod_{j=2}^{N} f(-j+1) \\
& =\prod_{j=2}^{N} f(-j+1) \prod_{j=2}^{N} f(j) \cdot[f(N+1)+f(-1-N)]=0,
\end{aligned}
$$

since $f$ is odd.

To calculate the remaining partials, we need only take the first term into account. Taking first $k=N$, the factor $f\left(w_{N}-N\right)$ yields a zero unless $l=N$. Thus, we get

$$
\begin{gathered}
\left(\partial_{l} F_{N}\right)\left(W_{0}\right)=0, \quad l=1, \ldots, N-1, \\
\left(\partial_{N} F_{N}\right)\left(W_{0}\right)=\prod_{j=1}^{N-1} f(N+1-j) \prod_{j=1}^{N} f(j+1)=f(N+1) \prod_{k=2}^{N} f(k)^{2} .
\end{gathered}
$$

Taking next $k<N$, we get a zero for $j=k+1$ unless the pertinent factor is differentiated. Hence, we obtain

$$
\begin{gathered}
\left(\partial_{l} F_{k}\right)\left(W_{0}\right)=0, \quad k<N, \quad l \neq k, k+1, \\
\left(\partial_{k+1} F_{k}\right)\left(W_{0}\right)=-f(k-N) \prod_{j \neq k, k+1} f(k-j+1) \prod_{j=1}^{N} f(j+1), \quad k<N, \\
\left(\partial_{k} F_{k}\right)\left(W_{0}\right)=-\left(\partial_{k+1} F_{k}\right)\left(W_{0}\right), \quad 1<k<N .
\end{gathered}
$$

Summarizing, the functional matrix is of the form

$$
(D F)\left(W_{0}\right)=\left(\begin{array}{cccccc}
0 & -a_{1} & 0 & \cdots & 0 & 0 \\
0 & a_{2} & -a_{2} & \cdots & 0 & 0 \\
\vdots & \vdots & \vdots & \ddots & \vdots & \vdots \\
0 & 0 & 0 & \cdots & a_{N-1} & -a_{N-1} \\
0 & 0 & 0 & \cdots & 0 & a_{N}
\end{array}\right)
$$

so its determinant vanishes.

From now on we assume (A6) holds true. Then we deduce that

$$
a_{2}, \ldots, a_{N} \neq 0
$$

so the principal minor with indices $2, \ldots, N$ is nonzero. Therefore, the implicit function theorem guarantees a solution to the system (A7) with the asserted properties. [Note that (A9) amounts to $w_{k}^{\prime}(0)=0, k=2, \ldots, N$; these relations follow from the explicit formula (A16) via implicit differentiation.]

For a general function $f$ the solution $W(t)$ to (A7) need not be a solution to (A3), i.e., one has $F_{1}(W(t)) \neq 0$ for $t$ near 0 . On the other hand, whenever $f$ is such that $F_{1}(W(t))=0$ for $t$ near 0 , one readily deduces from the inverse function theorem that one must have $|D F(W(t))|=0$ for $t$ near 0 . 


\section{APPENDIX B: UNIQUENESS OF JOINT EIGENFUNCTIONS}

As we have seen in Sec. IV, the joint eigenspace

$$
\mathcal{V}(y) \equiv\left\{M \in \mathcal{M} \mid B_{\delta} M=E_{\delta}(y) M, \delta=+,-\right\},
$$

with $\mathcal{M}$ given by (1.18), the $\mathrm{A} \Delta \mathrm{Os} B_{\delta}$ by (4.7), and their eigenvalues $E_{\delta}(y)$ by (3.20), contains the holomorphic functions $\mathcal{H}(x, y)$ and $\mathcal{H}(-x, y)$, and hence all of their linear combinations. But when $a_{+} / a_{-}$is rational, then $\mathcal{V}(y)$ is infinite-dimensional. Indeed, letting $a_{+}=p a$ and $a_{-}=q a$ with $p$ and $q$ coprime integers, all $m \in \mathcal{M}$ with period $i a$ have periods $i a_{+}$and $i a_{-}$, too. Thus, for any two multipliers $m_{+}, m_{-} \in \mathcal{M}$ with period $i a$, we have

$$
m_{+}(x) \mathcal{H}(x, y)+m_{-}(x) \mathcal{H}(-x, y) \in \mathcal{V}(y) .
$$

This entails $\operatorname{dim}(\mathcal{V}(y))=\infty$, as asserted.

By contrast, when $a_{+} / a_{-}$is irrational, then $\mathcal{V}(y)$ is 2-dimensional for $y$ sufficiently large. This is the content of Theorem B.1 below. As a preparation for this theorem and its proof we recall some well-known general features of the second order $\mathrm{A} \Delta \mathrm{Es}$ at issue in this paper; cf., for example, Nörlund's monograph. ${ }^{15}$

We start from an $\mathrm{A} \Delta \mathrm{E}$ of the form

$$
f_{+}(x) M(x+c)+f_{-}(x) M(x-c)=g(x) M(x), \quad c \in \mathbb{C}^{*},
$$

where $f_{+}, f_{-}, g \in \mathcal{M}$ with $f_{+}, f_{-} \neq 0$, and where only solutions $M \in \mathcal{M}$ are considered. Let $M_{1}, M_{2}$ be two solutions to (B3). Then the Casorati determinant,

$$
C\left(M_{1}, M_{2} ; x\right) \equiv M_{1}(x+c / 2) M_{2}(x-c / 2)-M_{1}(x-c / 2) M_{2}(x+c / 2),
$$

vanishes identically iff $M_{1} / M_{2}$ belongs to the field $F_{c}$ of $c$-periodic meromorphic functions. Assuming from now on $M_{1} / M_{2} \notin F_{c}$, the function (B4) is a solution to the first order $\mathrm{A} \Delta \mathrm{E}$

$$
\frac{C(x+c / 2)}{C(x-c / 2)}=\frac{f_{-}(x)}{f_{+}(x)},
$$

as is readily verified.

Next, assume $M_{3}(x)$ is a third solution to (B3). Then one easily verifies the identity

$$
M_{3}(x)=m_{1}(x) M_{2}(x)-m_{2}(x) M_{1}(x),
$$

with

$$
m_{j}(x) \equiv C\left(M_{j}, M_{3} ; x+c / 2\right) / C\left(M_{1}, M_{2} ; x+c / 2\right), \quad j=1,2 .
$$

Now quotients of Casorati determinants are $c$-periodic in view of the $\mathrm{A} \Delta \mathrm{E}$ (B5), so one has $m_{1}, m_{2} \in F_{c}$. Conversely, any function of the form (B6) with $m_{1}, m_{2} \in F_{c}$ solves (B3). Whenever two solutions exist whose Casorati determinant is not identically zero, the solution space is, therefore, 2-dimensional over the field $F_{c}$ of $c$-periodic meromorphic functions.

Consider now two $\mathrm{A} \Delta \mathrm{Es}$ of the form (B3), with shift parameters

$$
c_{1}=i a_{+}, \quad c_{2}=i a_{-}, \quad a_{+}, a_{-}>0 .
$$

Assume that two joint solutions exist whose Casorati determinants w.r.t. $c_{1}$ and $c_{2}$ are nonzero. When $a_{+} / a_{-} \in \mathbb{Q}$, then the joint solution space is infinite-dimensional, as we have already seen above. (Here and from now on, the field of scalars is again C.) It may well be that for $a_{+} / a_{-}$ irrational one can show that the assumptions just stated imply that the joint solution space is 2-dimensional, but we are not aware of a proof. 
For the concrete situation encountered in the main text, however, we have explicit solutions available. We shall now exploit this to prove 2-dimensionality for the case at hand.

Theorem B.1: Assume $a_{+} / a_{-} \notin \mathbb{Q}$. Then there exists $L \geqslant K$ such that for all $y \in(L, \infty)$ the joint eigenspace $\mathcal{V}(y)(B 1)$ is 2-dimensional with basis vectors $\mathcal{H}(x, y)$ and $\mathcal{H}(-x, y)$.

Proof: Consider the quotient function

$$
Q(x) \equiv \mathcal{H}(x, y) / \mathcal{H}(-x, y), \quad y \in(K, \infty) .
$$

In view of (3.39) and (3.5), it reads

$$
Q(x)=\prod_{\delta=+,-} \prod_{j=1}^{N_{\delta}} \frac{s_{-\delta}\left(x+z_{j}^{\delta}\right)}{s_{-\delta}\left(-x+z_{j}^{\delta}\right)} \cdot e^{2 x \Sigma}, \quad \Sigma=i r\left(2 N_{+} N_{-}+N_{+}+N_{-}+1\right)+i y .
$$

Since $z_{j}^{\delta} \in i(0, \infty)$, the function $Q(x)$ is analytic for $\operatorname{Re} x \in(0, \pi / r)$. We claim that there exists $L$ $\geqslant K$ such that for all $y \in(L, \infty)$ we have

$$
\lim _{\operatorname{Im} x \rightarrow \infty} Q(x)=0, \quad \operatorname{Re} x \in(0, \pi / r) .
$$

To prove this claim, we first note that the $\mathrm{A} \Delta \mathrm{E}(1.10)$ entails the bound

$$
\left|\frac{s(r, a ; x+z)}{s(r, a ; x-z)}\right| \leqslant C \exp \left(4 r|z| \frac{\operatorname{Im} x}{a}\right), \quad \operatorname{Re} x \in(0, \pi / r), \quad z \in i(0, \infty), \quad \operatorname{Im} x \rightarrow \infty .
$$

Therefore we have

$$
|Q(x)|=O(\exp [2(r \eta-y) \operatorname{Im} x]), \quad \operatorname{Re} x \in(0, \pi / r), \quad \operatorname{Im} x \rightarrow \infty,
$$

with

$$
\eta \equiv 2\left(\frac{1}{a_{+}} \sum_{j=1}^{N_{-}}\left|z_{j}^{-}\right|+\frac{1}{a_{-}} \sum_{j=1}^{N_{+}}\left|z_{j}^{+}\right|\right)-2 N_{+} N_{-}-N_{+}-N_{-}-1 .
$$

Now the sums have finite limits as $y \rightarrow \infty$ [recall (3.14)], so there exists $L \geqslant K$ such that $r \eta<y$ for all $y \in(L, \infty)$. Hence, our claim follows.

Fixing $y \in(L, \infty)$, it now follows from (B11) that $Q(x)$ is neither $i a_{+}$-periodic nor $i a_{-}$-periodic. Therefore, the Casorati determinants of $\mathcal{H}(x, y)$ and $\mathcal{H}(-x, y)$ w.r.t. $i a_{+}$and $i a_{-}$ are nonzero. Letting $M(x) \in \mathcal{V}(y)(\mathrm{B} 1)$, we then have both

$$
M(x)=\lambda_{+}(x) \mathcal{H}(x, y)+\lambda_{-}(x) \mathcal{H}(-x, y),
$$

with $\lambda_{+}, \lambda_{-} \in F_{i a_{+}}$, and

$$
M(x)=\mu_{+}(x) \mathcal{H}(x, y)+\mu_{-}(x) \mathcal{H}(-x, y),
$$

with $\mu_{+}, \mu_{-} \in F_{i a_{-}}$.

Next, we combine (B15) and (B16) to obtain

$$
\lambda_{-}(x)-\mu_{-}(x)=\left(\mu_{+}(x)-\lambda_{+}(x)\right) Q(x) .
$$

Since $\lambda_{+}(x)$ and $\lambda_{-}(x)$ are $i a_{+}$-periodic meromorphic functions, they are analytic on the lines $\operatorname{Re} x=\rho \in[0, \pi / r]$, save for finitely many $\rho$. Similarly, $\mu_{+}(x)$ and $\mu_{-}(x)$ have this property. Now let $\rho_{0} \in(0, \pi / r)$ be such that $\lambda_{+}, \lambda_{-}, \mu_{+}$, and $\mu_{-}$are analytic on $\operatorname{Re} x=\rho_{0}$. By periodicity, $\lambda_{+}$ and $\mu_{+}$are bounded on this line, so (B11) and (B17) entail 


$$
\lim _{\operatorname{Im} x \rightarrow \infty}\left(\lambda_{-}(x)-\mu_{-}(x)\right)=0, \quad \operatorname{Re} x=\rho_{0} \in(0, \pi / r) .
$$

In particular, this implies that

$$
\lim _{k \rightarrow \infty} \lambda_{-}\left(\rho_{0}+i k a_{-}\right)=\mu_{-}\left(\rho_{0}\right), \quad k \in \mathbb{N} .
$$

Thus far, we have not used our assumption that $a_{+} / a_{-}$is irrational. But now we can combine this assumption with (B19) to deduce that $\lambda_{-}(x)$ equals $\mu_{-}\left(\rho_{0}\right)$ for $\operatorname{Re} x=\rho_{0}$ and so for all $x$. [Indeed, the numbers $\rho_{0}+i k a_{-}, k>N$, are dense $\left(\bmod i a_{+}\right)$in the interval $\rho_{0}+i\left[0, a_{+}\right)$for

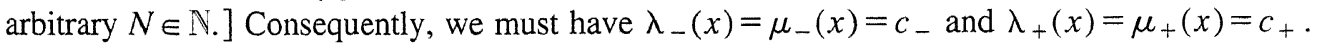

'S. N. M. Ruijsenaars, "Complete integrability of relativistic Calogero-Moser systems and elliptic function identities," Commun. Math. Phys. 110, 191-213 (1987).

${ }^{2}$ S. N. M. Ruijsenaars, "Finite-dimensional soliton systems," in Integrable and Superintegrable Systems, edited by B. Kupershmidt (World Scientific, Singapore, 1990), pp. 165-206.

${ }^{3}$ S. N. M. Ruijsenaars, "Systems of Calogero-Moser type," to be published in Proceedings of the 1994 Banff Summer School 'Particles and fields,' edited by G. Semenoff and L. Vinet.

${ }^{4}$ E. T. Whittaker and G. N. Watson, A Course of Modern Analysis (Cambridge University Press, Cambridge, 1973).

${ }^{5}$ E. K. Sklyanin, "Some algebraic structures connected with the Yang-Baxter equation,'” Funct. Anal. Appl. 16, 27-34 (1982).

${ }^{6}$ E. K. Sklyanin, "Some algebraic structures connected with the Yang-Baxter equation. Representations of quantum algebras," Funct. Anal. Appl. 17, 273-284 (1983).

${ }^{7}$ I. Krichever and A. Zabrodin, "Spin generalization of the Ruijsenaars-Schneider model, non-Abelian 2D Toda chain and representations of Sklyanin algebra," Russ. Math. Surveys 50, 1101-1150 (1995).

${ }^{8} \mathrm{G}$. Felder and A. Varchenko, "Algebraic Bethe Ansatz for the elliptic quantum group $E_{\tau, \eta}\left(s l_{2}\right)$," Nucl. Phys. B 480, 485-503 (1996).

${ }^{9}$ G. Felder and A. Varchenko, "Algebraic integrability of the two-body Ruijsenaars operator," preprint.

${ }^{10} \mathrm{~K}$. Hasegawa, "Ruijsenaars' commuting difference operators as commuting transfer matrices," Commun. Math. Phys. 187, 289-325 (1997).

${ }^{11}$ S. N. M. Ruijsenaars, "Generalized Lamé functions. II. Hyperbolic and trigonometric specializations,' J. Math. Phys. 40, 1627-1663 (1999).

${ }^{12}$ S. N. M. Ruijsenaars, "Relativistic Lamé functions: The special case $g=2$," to appear in J. Phys. A.

${ }^{13}$ S. N. M. Ruijsenaars, "On relativistic Lamé functions," to be published in Proceedings of the 1997 Montreal Workshop on Calogero-Moser-Sutherland systems, edited by J. F. van Diejen and L. Vinet.

${ }^{14}$ S. N. M. Ruijsenaars, "First order analytic difference equations and integrable quantum systems," J. Math. Phys. 38, 1069-1146 (1997).

${ }^{15}$ N. E. Nörlund, Vorlesungen über Differenzenrechnung (Springer-Verlag, Berlin, 1924). 